\title{
A screening for suppressor mutants reveals components involved in the blue light-inhibited sexual filamentation in Cryptococcus neoformans
}

\author{
Yu-Ling Yeh ${ }^{1}$, Yu-Sheng Lin ${ }^{1}$, Bei-Jia Su, Wei-Chiang Shen ${ }^{*}$ \\ Department of Plant Pathology and Microbiology, National Taiwan University, Room 216, Building Number 1, No. 1 Roosevelt Road, Sec. 4, 10617 Taipei, Taiwan
}

\section{A R T I C L E I N F O}

\section{Article history:}

Received 17 June 2008

Accepted 20 October 2008

Available online 25 October 2008

\section{Keywords:}

Cryptococcus neoformans

Photoresponse

Cwc1

Cwc2

Agrobacterium insertional mutagenesis

Suppressor

\begin{abstract}
A B S T R A C T
Blue light regulates diverse physiological and developmental processes in fungi. Our prior studies demonstrated that the evolutionally conserved $\mathrm{Cwc} 1$ and $\mathrm{CwC} 2$ proteins mediate the blue light-inhibited sexual filamentation in Cryptococcus neoformans. To characterize the putative domains of the Cwc1 and Cwc2 proteins, we generated partially deleted versions of these genes under the GPD1 promoter and examined their effects. The results confirmed that LOV and PAS domains are essential for the function of the Cwc1 protein, and the PAS domain and zinc finger DNA-binding motif are also crucial for the Cwc2 protein. To further understand how light inhibits filamentous growth, a genome wide mutant screening was conducted to identify genes important for this process. Mutants which suppressed the light-dependent CWC1 overexpression phenotype and restored mating filamentation were identified. In the one with fully restored filamentation, the T-DNA was found to disrupt the expression of the CWC2 gene. Additionally, a mediator component, the SSN8 gene, known to involve in transcriptional regulation was also identified. Our results demonstrate that Cwc1 and Cwc2 are two central regulators of the C. neoformans photoresponses and the roles of other components identified in the screen are under investigation.
\end{abstract}

(c) 2008 Elsevier Inc. All rights reserved.

\section{Introduction}

Ambient environmental cues such as light are crucial for diverse organisms including filamentous fungi. Growth and development of many fungal species are intricately regulated by light (Carlile, 1965; Purschwitz et al., 2006; Tan, 1978). Fungal responsiveness to different wavelengths of light has been well documented, and blue light/UV and red/far-red light are two types of photoresponses primarily observed (Griffith et al., 1994; Mooney and Yager, 1990; Purschwitz et al., 2006). Although photoreceptors for different action spectra are present in individual fungal species, only one type of photoresponses was predominantly seen (Blumenstein et al., 2005; Froehlich et al., 2002,2005; He et al., 2002; Purschwitz et al., 2008). As such, blue light photoresponses have been most widely observed in fungal kingdom and were subjected to intensive studies in recent years (Herrera-Estrella and Horwitz, 2007; Purschwitz et al., 2006).

In fungi, Neurospora crassa has been the primary model for studying fungal blue light photobiology (Linden et al., 1997). Two central regulators, $w c-1$ and $w c-2$, were identified as mutants with altered carotenoid pigment production in response to light, and other light responsive behaviors in these mutants were also defective (Ballario et al., 1996; Linden and Macino, 1997). The WC-1 protein functions as the FAD-binding photoreceptor (Froehlich et al.,

\footnotetext{
* Corresponding author. Fax: +886223636490.

E-mail address: wcshen@ntu.edu.tw (W.-C. Shen).

1 These authors contributed equally to this work.
}

2002; He et al., 2002) and transcription factor to interact with WC-2 to coordinately regulate genes involved in the pigmentation, differentiation, and circadian rhythm (Linden et al., 1997; Liu et al., 2003). Recent studies revealed that post-translational modification of WC-1 and WC-2 is critical for their regulation. WC proteins are phosphorylated in the dark and also in a light-dependent manner. The degree of their phosphorylation status is important for their binding activity (Dunlap and Loros, 2006). Light triggers the binding of L-WCC (Light WC-1/WC-2 complexes) to the LREs (light response elements) of immediate early light responsive genes such as frq, al-3, and $v v d$ and activates their expression (He and Liu, 2005). Binding of L-WCC to the LREs can only be transient and hyperphosphorylation of WCC inhibits WC activity, resulting in the repression of frq transcription (He et al., 2006). Phosphorylation has been shown to occur sequentially in WC-1 phosphorylation sites. The cAMP-dependent protein kinase A first acts as a FRQ-independent priming kinase (Huang et al., 2007) and then FRQ-dependent hyperphosphorylation mediated by casein kinases CKII and CK-1a in the light eventually leads to the binding inhibition of the L-WCC to the LREs (He et al., 2006; Huang et al., 2007). Additionally, acetylation of histone $\mathrm{H} 3$ lysine 14 associated with the promoter of al-3 was also demonstrated to be required for the WC-1-dependent blue light response (Grimaldi et al., 2006). These studies provide the detailed molecular mechanisms from light-induced transcription to photoadaptation in N. crassa.

Since the initial finding and detailed characterization in $N$. crassa, homologues of $w c-1$ and $w c-2$ were also identified by bioinformatic and genetic approaches in fungi across different phyla 
(Corrochano, 2007). Examples of the $w c-1$ orthologues in the Zygomycetes include the madA and $w c o A$ genes of Phycomyces blakesleeanus (Idnurm et al., 2006), the mcwc-1a, mcwc-1b, and mcwc-1c genes of Mucor circinelloides (Silva et al., 2006). Orthologues of wc-1 in several Ascomycetes include the blr-1 gene of Trichoderma atroviride (Casas-Flores et al., 2004), the Tbwc-1 gene of Tuber borchii (Ambra et al., 2004), the mgwc-1 gene of Magnaporthe grisea (Lee et al., 2006), the BLR1 gene of Bipolaris oryzae (Kihara et al., 2007), and the wcoA gene of Fusarium fujikuroi (Estrada and Avalos, 2008). In the Basidiomycetes, the dst1 gene of Coprinus cinereus (Terashima et al., 2005), and the BWC1/CWC1 gene of Cryptococcus neoformans (Idnurm and Heitman, 2005; Lu et al., 2005) have also been characterized.

Cryptococcus neoformans is an important human fungal pathogen with a well-defined life cycle (Casadevall and Perfect, 1998; Lin and Heitman, 2006). This fungus has become a model for studying fungal virulence, physiology, and differentiation due to its wellestablished molecular and genomic resources (Hull and Heitman, 2002; Loftus et al., 2005). C. neoformans grows vegetatively as yeasts under normal growth conditions, and filamentous hyphae develop in the sexual differentiation process. $C$. neoformans is a heterothallic basidiomycete with MAT $\alpha$ and MATa mating types. Two types of sexual development, mating and monokaryotic fruiting, have been described (Kwon-Chung, 1976; Lin et al., 2005). Mating in C. neoformans is a sexual process involved two opposite mating type cells as typically seen in the heterothallic fungi. Fusion of the conjugated structures produced by the haploid yeast cells leads to the production of dikaryotic hyphae, which give rise to the robustly filamentous appearance of the mating colony. Nuclear fusion and meiosis occur at the tips of hyphae to subsequently generate four chains of sessile basidiospores (Kwon-Chung, 1976). In contrast, monokaryotic fruiting is a differentiation process primarily associated with the MATa cells (Wickes et al., 1996). Under nitrogen starvation and desiccation conditions, rare cell fusion or endoduplication events have been speculated to occur to form the diploid cells. Diploid cells further undergo filamentation process to generate the monokaryotic filaments, which show sparse filamentation around the colony (Lin et al., 2005). Components such as pheromones and pheromone receptors required for $\alpha$ and a cell fusion in mating were also shown to function before cell or nuclear fusion during monokaryotic fruiting process (Lin et al., 2005; Shen et al., 2002).

Reports from our laboratory and others showed that filamentation associated with mating and monokaryotic fruiting is inhibited specifically by blue light (Idnurm and Heitman, 2005; Lu et al., 2005). C. neoformans orthologues of $N$. crassa WC- 1 and WC- 2 were identified and named independently as Cwc1/Bwc1 and Cwc2/ Bwc2 (Cwc, Cryptococcus white collar; Bwc, Basidiomycete white collar). Conserved LOV (for Light-Oxygen-Voltage), PAS (for PerArnt-Sim), and GATA-type zinc finger DNA-binding domains are found among these proteins ( $\mathrm{Lu}$ et al., 2005). Deletion studies showed that both of these genes are crucial for mediating lightinhibition of mating and monokaryotic filamentation (Idnurm and Heitman, 2005; Lu et al., 2005). Overexpression of these genes further inhibits filamentation upon light treatment (Lu et al., 2005). Interestingly, these genes also contribute to UV resistance and full virulence of $C$. neoformans (Idnurm and Heitman, 2005).

The goals of our study are to dissect the protein regions important for the functions of Cwc1 and Cwc2 and to understand how light regulates filamentous growth. In this report, we describe the characterization of putative domains identified in the Cwc1 and Cwc2 proteins. Using genetic approach, we also demonstrate that the presence of functional copies of both $C W C 1$ and $C W C 2$ genes is required for proper light response. To address the issue of how light inhibits filamentation via the Cwc proteins, we have conducted a screen for suppressors of the CWC1 overexpression strain using the Agrobacterium-mediated insertional mutagenesis technique. In this study, we report the identification of the CWC2 and SSN8 genes whose mutation suppressed the light-dependent CWC1 overexpression phenotype.

\section{Materials and methods}

\subsection{Strains, media, and growth conditions}

Cryptococcus neoformans strains used in this study are listed in Table 1. Congenic serotype D strains JEC20 (MATa) and JEC21 (MA$T \alpha$ and their auxotrophic derivatives were used throughout the study (Edman and Kwon-Chung, 1990; Moore and Edman, 1993). All strains were handled using standard techniques and media as previously described (Alspaugh et al., 1997; Guthrie and Fink, 1991). Yeast extract-peptone-dextrose (YPD) medium, synthetic (SD) medium, V8 agar, and filament agar were prepared as described (Alspaugh et al., 1997; Wickes et al., 1996). C. neoformans strains were routinely maintained on YPD at $30^{\circ} \mathrm{C}$, and mating was conducted on V8 agar at $26^{\circ} \mathrm{C}$.

\subsection{Construction of the truncated Cwc1 proteins}

To construct the truncated versions of the $C$. neoformans Cwc1 proteins, overlapping PCR with the primers flanking the deleted region was conducted and the PCR product was cloned into pYKL8 under the control of GPD1 (glyceraldehyde-3-phosphate dehydrogenase gene 1) promoter (Lu et al., 2005). To make the LOV domain-deleted CwC1 protein, two sets of PCR primers flanking the LOV domain were designed and PCR amplification was conducted with the previously reported CWC1 overexpression plasmid pYKL9 as the DNA template (Lu et al., 2005). All the oligonucleotide primers used in this study are listed in Table 2. The $5^{\prime}$-flanking $1.76 \mathrm{~kb}$ and $3^{\prime}$-flanking $1.93 \mathrm{~kb}$ fragments of the LOV domain region were amplified with WC241/WC346 and WC347/

Table 1

C. neoformans strains used in this study.

\begin{tabular}{|c|c|c|}
\hline Strain & Description & Reference \\
\hline JEC20 & MATa & Kwon-Chung et al. (1992) \\
\hline JEC21 & 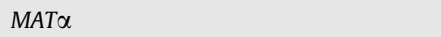 & Kwon-Chung et al. (1992) \\
\hline JEC34 & MATa ura5 & Moore and Edman (1993) \\
\hline JEC43 & MAT $\propto$ ura5 & Moore and Edman (1993) \\
\hline H99 & 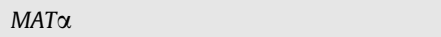 & Wang et al. (2000) \\
\hline $\mathrm{BJC2}$ & 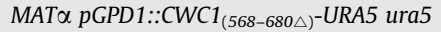 & This study \\
\hline BJC5 & MAT $\alpha$ cwc1 + pGPD1::CWC1-URA5 ura5 & This study \\
\hline BJC6 & $\begin{array}{l}\text { MAT } \alpha \text { cWc1 } 1+p G P D 1:: C W C 1_{(568-680 \triangle)^{-}} \\
\text {URA5 ura5 }\end{array}$ & This study \\
\hline BJC7 & MAT $\alpha$ pGPD1::CWC1 $1_{(767-929 \triangle)}$-URA5 ura5 & This study \\
\hline BJC8 & $\begin{array}{l}\text { MAT } \alpha \text { cWc1 }+p G P D 1:: C W C 1_{(767-929 \triangle)^{-}} \\
\text {URA5 ura5 }\end{array}$ & This study \\
\hline $\mathrm{KHC2}$ & MAT $\alpha$ cwc2::URA5 ura5 & This study \\
\hline KHC4 & MAT $\alpha$ cwc2 $\operatorname{ura5}\left(5-\mathrm{FOA}^{\mathrm{r}}\right)$ & This study \\
\hline KHC6 & MAT $\alpha$ pGPD1::CWC2-URA5 ura5 & Lu et al. (2005) \\
\hline КHC21 & MAT $\alpha$ cWc1 + pGPD1::CWC2-URA5 ura5 & This study \\
\hline YKC7 & MAT $\alpha$ cwc1::URA5 ura5 & Lu et al. (2005) \\
\hline YKC25 & MAT $\alpha$ ura5 cwc1 $\left(5-\mathrm{FOA}^{\mathrm{r}}\right)$ & Lu et al. (2005) \\
\hline YКС38 & MAT $\alpha$ pGPD1::CWC1-URA5 ura5 & Lu et al. (2005) \\
\hline YLC2 & MAT $\alpha$ cWc2 + pGPD1::CWC1-URA5 ura5 & This study \\
\hline YLC3 & MAT $\alpha$ cWc2 + pGPD1::CWC2-URA5 ura5 & This study \\
\hline YLC5 & $M A T \alpha c w c 1 c w c 2$ & This study \\
\hline YLC9 & MAT $\alpha$ pGPD1::CWC2 ${ }_{(72-140 \triangle}-$ URA5 ura5 & This study \\
\hline YLC12 & $\begin{array}{l}\text { MAT } \alpha \text { cWc2 }+ \text { pGPD1::CWC2 }(72-140 \triangle)^{-} \\
\text {URA5 ura5 }\end{array}$ & This study \\
\hline YLC18 & MAT $\alpha$ pGPD1::CWC2 ${ }_{(349-373 \triangle)^{-}}$URA5 ura5 & This study \\
\hline YLC19 & $\begin{array}{l}\text { MAT } \alpha \text { cWc2 }+p G P D 1:: C W C 2_{(349-373 \triangle)^{-}} \\
\text {URA5 ura5 }\end{array}$ & This study \\
\hline YSC1 & MAT $\alpha$ ssn $8+p G P D 1:: C W C 1-U R A 5$ ura5 & This study \\
\hline YSC13-1 9 & Cross progeny of AY18 and JEC20 & This study \\
\hline
\end{tabular}


Table 2

PCR primers used in this study.

\begin{tabular}{ll}
\hline Name & Sequence $\left(5^{\prime}-3^{\prime}\right)$ \\
\hline WC53 & CCAGAGCGCATCGTGAGCATT \\
WC54 & CTCACCCACTACGATCGTCGT \\
WC64 & CGTGTGTTCTTGGAATGGCT \\
WC65 & GTCTCCGAGCCGAATTTTCA \\
WC241 & CGGGATCCATGTCAACAAACCTCACTTCT \\
WC325 & CGGGATCCAATGTCCCTCCTCGCCGAGTCT \\
WC326 & TCCCCCGGGAAGGCCGAAATGAAATAAGCGT \\
WC346 & ACTACAACTCAGGTCGACAGGTCC \\
WC347 & TGGACCTGTCGACCTGAGTTGTAGTGTTGAACAGCCAAACAAAATCCTCCGT \\
WC355 & TCCCCCGGGTAGTTTAGAACCTGTTCCCT \\
WC373 & GTGCCCTGTCGCTTGCGTCATCGT \\
WC402 & GAGTGATAAG GCATGTATGAAGTCT \\
WC403 & AGACTTCATACATGCCTTATCACTCCCGTCTGGCGAACATCTCATTAATA \\
WC406 & GATTGCGTTCCCGCCTTCA \\
WC407 & CGTCCGCAATGTGTTATTAAG \\
WC408 & GTCTCTGAAACCAGGAAGCTA \\
WC409 & GCATGTTATGGAGTCCTC \\
WC419 & TGGCGCACGATTTGCGGAAAGGAA \\
WC433 & ACTCCAAGCTTCGCCGTTCTGATA \\
WC434 & TATCAGAACGGCGAAGCTTGGAGTCGGACAACATACATCAGGATGCTCT \\
WC435 & GCAAACATGCATTGTCTCTCCCTCT \\
WC436 & AGAGGGAGAGACAATGCATGTTTGCGGACTAAGATGGGCAAAACGAAAT \\
WC445 & CGGGATCCATGTCTTCCAACTTCTATACCTCCT \\
WC446 & TCCCCCGGGCCACTGCCAGATACTTCTCCGAA \\
WC466 & CCAGCTCTCAACGCATTTACAAGCA \\
WC467 & CGGGATCCTCTTTGGTCAATTGGGCAGATGG \\
WC468 & CGGGATCCATCGCATGCGTATATGTACACT \\
WC469 & CGTTACTGGTCGAGACGTTCAGTT \\
WC496 & CATAATGTCGACTGTTGGACAG \\
WC498 & GGGGTACCAATGGTGCACTATGGTTGGT \\
& \\
&
\end{tabular}

WC355, respectively. The PCR products were purified and at least $40 \mathrm{ng}$ of each PCR product was mixed in equal molar ratio and further amplified with WC241/WC335 to obtain the final $3.69 \mathrm{~kb}$ PCR product. The product was then purified, restriction digested with BamHI and SmaI, and ligated into pYKL8 to obtain pBJS12. The construct was sequenced to verify no additional changes. The LOV domain truncated version of the CWC1 construct pBJS12 was then biolistically transformed into the MATa ura5 strain JEC43 and the MAT $\alpha$ ura5 cwc1 mutant strain YKC25 (Lu et al., 2005). Uracil prototrophic transformants were picked and screened by PCR. Since the construct was not targeted to a specific locus and integration might occur ectopically at different sites among transformants. We further verified the expression level of the transgene by Northern blot analysis and transformants with high and similar transcript level were selected for phenotypic characterization.

To make the PAS domains-deleted Cwc1 protein, two sets of PCR primers flanking the PAS domains were also designed and PCR amplification was conducted with the plasmid pYKL9 (Lu et al., 2005). The 5'-flanking $2.46 \mathrm{~kb}$ and $3^{\prime}$-flanking $1.13 \mathrm{~kb}$ fragments of the PAS domains region were amplified with WC241/ WC402 and WC403/WC355, respectively. An equal molar ratio of at least $40 \mathrm{ng}$ purified PCR products were further used to amplify with WC241/WC335 to obtain the final $3.59 \mathrm{~kb}$ PCR product. The product was similarly cloned into pYKL8 to obtain pBJS13 and no other mutation was also confirmed by sequencing. The PAS domains truncated version of the CWC1 construct pBJS13 was transformed into the MAT $\alpha$ ura5 strain JEC43 and the MAT $\alpha$ ura5 cwc1 mutant strain YKC25. Uracil prototrophic transformants were picked and screened by PCR and the expression level was also verified by Northern blot analysis.

\subsection{Construction of the truncated Cwc2 proteins}

To make the truncated versions of the $C$. neoformans $\mathrm{Cwc} 2$ proteins, we also conducted overlapping PCR and similarly expressed the products under the GPD1 promoter. To make the PAS domaindeleted Cwc2 protein, two sets of PCR primers flanking the PAS domain were designed and PCR amplification was conducted with the plasmid pKHS1 (Lu et al., 2005). The 5'-flanking $0.3 \mathrm{~kb}$ and 3'-flanking $1.6 \mathrm{~kb}$ fragments of the PAS domain region were amplified with WC325/WC433 and WC434/WC326, respectively. The PCR products were purified, mixed, and used as a template for further amplification with WC325/WC326 to obtain the final $1.9 \mathrm{~kb}$ PCR product. The product was further purified, digested with BamHI and SmaI, and ligated with pYKL8 to obtain pYLY1. The PAS domain truncated version of the CWC2 construct PYLY1 was verified by sequencing and biolistically transformed into the MAT $\alpha$ ura5 strain JEC43 and the MAT $\alpha$ ura5 cwc2 mutant strain KHC4. Uracil prototrophic transformants were picked, screened by PCR, and verified by Northern blot analysis.

To make the $C W C 2$ gene without the zinc finger DNA-binding domain, PCR primers WC325/WC435 and WC326/WC436 were designed to amplify the 5 - and $3^{\prime}$-flanking sequences, respectively. The amplified 1.25 and $0.77 \mathrm{~kb}$ PCR fragments were purified and mixed for overlapping PCR with primers WC325 and WC326 to obtain the final PCR product. The product was cloned into pYKL8 and the sequence of the resulting pYLY6 was confirmed no additional mutation and transformed into the MAT $\alpha$ ura5 strain JEC43 and the MATa ura 5 cwc2 mutant strain KHC4. Uracil prototrophic transformants were picked, screened by PCR, and verified by Northern blot analysis.

\subsection{Overexpression of the CWC1 gene in the cwc2 mutant background} and overexpression of the CWC2 gene in the cwc1 mutant background

To overexpress the CWC1 gene in the $c w c 2$ mutant, pYKL9 was utilized to transform the $c w c 2$ 5-FOA selected strain KHC4. The resulting uracil prototrophic transformants were picked, screened by PCR, and verified by Northern blot analysis. To overexpress the $C W C 2$ gene in the $c w c 1$ mutant, pKHS1 was used to transform the $c w c 15$-FOA selected strain YKC25. The resulting uracil prototrophic transformants were similarly screened and verified by Northern blot analysis.

\subsection{Generation of the cwc1 cwc2 double mutants}

To generate the $c w c 1 \quad c w c 2$ double mutant strains, the cwc1::URA5 disruption allele (Lu et al., 2005) was introduced into the MAT $\alpha$ cwc2 ura5 (5-FOA ${ }^{\mathrm{r}}$ ) strain KHC4 by biolistic transformation. The resulting uracil prototrophic transformants were selected, screened by PCR, and verified by Southern blot analysis.

\subsection{Agrobacterium-mediated insertional mutagenesis and screening for suppressor mutants}

To conduct the insertional mutagenesis, Agrobacterium tumefaciens strain LBA4404 bearing the plasmid pPZP-201BK with NAT cassette was utilized for transformation (Idnurm et al., 2004). C. neoformans CWC1 overexpression strain YKC38 was targeted for mutant screening (Lu et al., 2005). In brief, A. tumefaciens strain was first grown in Luria-Bertani liquid medium supplemented with $50 \mu \mathrm{g} / \mathrm{ml}$ of kanamycin for 2 days at $28{ }^{\circ} \mathrm{C}$. Cells was then collected, washed with sterile water, and resuspended in induction medium with $100 \mu \mathrm{M}$ acetosyringone to an optical density of 0.15 at $660 \mathrm{~nm}$. The culture was further incubated for $6 \mathrm{~h}$ at $28^{\circ} \mathrm{C}$, and the optical density was expected to reach 0.6. C. neoformans strain YKC38 was grown in YPD liquid medium for overnight, washed with sterile water, and resuspended with induction medium to $10^{6}-10^{7}$ cells per ml. Each of A. tumefaciens and C. neoformans $(200 \mu \mathrm{l})$ cells were mixed and co-incubated on induction agar medium with $100 \mu \mathrm{M}$ acetosyringone for $48 \mathrm{~h}$, and cells were 
harvested and selected onto YPD agar medium with nourseothricin and cefotaxime each at the concentration of $100 \mu \mathrm{g} / \mathrm{ml}$.

C. neoformans transformants resistant to nourseothricin were streaked onto $0.1 \times$ YPD agar medium (with $2 \%$ glucose) for single colony isolation. Single colonies were picked and reselected on YPD medium with $100 \mu \mathrm{g} / \mathrm{ml}$ of nourseothricin. Strains maintaining the resistance were examined for mating filamentation by crossing with the MATa wild-type strain JEC20 on V8 medium at $26{ }^{\circ} \mathrm{C}$ in the presence of constant white light. Most strains were expected to display complete inhibition of sexual filamentation in the light as the original CWC1 overexpression strain. Strains restored for filament formation were categorized based on the level of filamentation and subjected to further characterization.

\subsection{Nucleic acid manipulations}

Agrobacterium T-DNA integration was confirmed by Southern blot analysis. Genomic DNA used for Southern hybridization was prepared by a large-scale isolation protocol described previously (Perfect et al., 1993). Hybridization probe NAT was prepared from pCH233 digested with EcoRI and KpnI and labeled by Prime-It ${ }^{\circledR}$ II Random Primer Labeling Kit (Stratagene) with $\left[\alpha-{ }^{32}\right.$ P]dCTP (NEN Life Science Products). To identify the T-DNA integration site, restriction enzymes which do not cut or cut once in the T-DNA vector were chosen to digest the genomic DNA prepared from the strain of interest. DNA was then purified, diluted, and self-ligated. Inverse PCR was subsequently conducted with primers WC406, WC407, WC408, and WC409, which reside in the T-DNA region, to recover the flanking sequences. PCR products with expected sizes based on Southern analysis were further purified, sequenced, and searched against the JEC21 genome and NCBI database to define the site of insertion and the gene of interest. All nucleic acid manipulations were performed according to standard procedures (Sambrook and Russell, 2001).

\subsection{Isolation and characterization of the progeny from the cross between the AY18 insertional mutant and MATa wild-type strain}

To obtain the progeny from the cross of AY18 and MATa wildtype strains, we first grew them on YPD at $30^{\circ} \mathrm{C}$ for 1 day. Equal amount of cells for both strains were separately resuspended in sterile water and mixed together. Before spotting the mating mixture, a metal ring of $1 \mathrm{~cm}$ in diameter and height was sterilized by flame, and pressed down on the surface of V8 agar to create an isolated circle area with $0.1 \mathrm{~cm}$ gap. Then the mixture was spotted onto the center of circle area and incubated in the dark for 10-14 days. When filaments crossed the gap and developed fruiting structures, the agar outside the gap was excised, put into $1.5 \mathrm{ml}$ of sterile water in a microfuge tube and vortexed to disperse the basidiospores. The suspension was spread onto YPD agar medium containing $100 \mu \mathrm{g} / \mathrm{ml}$ nourseothricin. The resistant transformants were streaked onto $0.1 \times$ YPD agar medium for single colony isolation. Colonies were picked and reselected on YPD medium with $100 \mu \mathrm{g} / \mathrm{ml}$ of nourseothricin. Stably resistant strains were subjected to further phenotypic and genotypic characterization.

To verify the genotypes of the progeny, genomic DNA was extracted and subjected to PCR and Southern blot hybridization. To confirm mating type, two sets of mating type specific PCR primers WC53/WC54 and WC64/WC65 were designed for the STE12 $\alpha$ and STE12a genes, respectively. The presence of intact $P_{G P D 1}-C W C 1$ expression cassette was confirmed by primer WC373 and WC419. T-DNA integration at the SSN8 locus in the AY18 mutant was confirmed by PCR primer WC407 and WC466. Uracil auxotrophy or prototrophy was determined by the growth on SD medium lacking or containing uracil.

\subsection{Disruption of the C. neoformans SSN8 gene in the CWC1 overexpression strain}

To disrupt the $C$. neoformans SSN8 gene in the CWC1 overexpression strain, a ssn8::NAT disruption allele was constructed for homologous recombination. Primer pairs of WC458/WC467 and WC468/WC469 were designed to amplify the flanking sequences of the SSN8 genomic region. The $1.2 \mathrm{~kb} 5^{\prime}$ - and 3'-flanking PCR products were, respectively, digested with SacI/BamHI and Bam$\mathrm{HI} / \mathrm{Xhol}$ and subcloned into pBluescript SK(+). Then four fragments ligation was conducted with the digested $5^{\prime}$ - and 3 '-flanking fragments, BamHI digested NAT selectable marker, and SacI/Xhol digested pBluescript. The resulting ssn8::NAT disruption allele was introduced into the YKC38 strain by biolistic transformation (Toffaletti et al., 1993). Transformants were selected on YPD medium with $100 \mu \mathrm{g} / \mathrm{ml}$ nourseothricin and verified by PCR and Southern blot analysis.

\subsection{RNA isolation and Northern blot analysis}

RNA used in Northern analysis was isolated from yeast cells by using the TRIzol total RNA isolation reagent according to the manufacturer's instructions (Invitrogen). Twenty micrograms of total RNA from each sample was separated by electrophoresis in a $1.2 \%$ agarose-formaldehyde gel. RNA was transferred by capillary action to nylon membrane (Immobilon ${ }^{\mathrm{TM}}-\mathrm{Ny}^{+}$, Millipore) and hybridized in hybridization buffer $\left(0.12 \mathrm{M} \mathrm{Na}_{2} \mathrm{HPO}_{4}\right.$ [pH 7.2 ], $0.25 \mathrm{M} \mathrm{NaCl}, 1 \mathrm{mM}$ EDTA [pH 8], 7\% SDS, and 50\% formamide). All probes were labeled as described for Southern blot analysis.

\subsection{Mating assay}

Strains for mating assay were first grown on YPD at $30^{\circ} \mathrm{C}$ for 1 day. Mating reactions were conducted by co-incubating cells with desired partners on V8 agar medium at $26^{\circ} \mathrm{C}$ under white light or dark conditions for 1-5 days. JEC20 (MATa) and JEC21 (MA$T \propto$ strains were used as mating testers. Dikaryotic filaments at the periphery of mating spot were evaluated under Olympus BX41 microscope. Pictures were taken $16-48 \mathrm{~h}$ post-incubation at $100 \times$ magnification.

\subsection{Monokaryotic fruiting assay}

Strains for monokaryotic fruiting assay were first grown on YPD at $30^{\circ} \mathrm{C}$ for 1 day. Yeast cells of each strain were suspended in sterile water and then $5 \mu \mathrm{l}$ was spotted on FA plate and incubated at $26{ }^{\circ} \mathrm{C}$ under white light or dark conditions for 3-14 days. Filaments at the periphery of the spot were evaluated under Olympus BX41 microscope. Pictures were taken 5-14 days post-incubation at $100 \times$ magnification.

\section{Results}

3.1. LOV and PAS domains of the Cwc1 protein are essential for blue light responses in $\mathrm{C}$. neoformans

Based on the predicted structural organization, a putative LOV domain, two putative PAS domains, and a putative nuclear localization sequence were identified in the $C$. neoformans Cwc1 protein. LOV domain was known as a specialized PAS domain responsible for chromophore binding and light sensing. Cwc1 has been proposed to function as a blue light photoreceptor through its putative FAD-binding LOV domain and light treatment was shown to be required for its activation (Lu et al., 2005). Cwc1 forms complex with Cwc2 possibly via the PAS domains and in turn binds to target gene promoter through the zinc finger DNA-binding domain of Cwc2 
and negatively regulates filamentous growth (Idnurm and Heitman, 2005; Lu et al., 2005).

To confirm the functions of the putative LOV and PAS domains, we generated truncated versions of the $C W C 1$ gene under the GPD1 promoter (Fig. 1A). The constructs were sequenced to confirm no additional mutation and were transformed into the C. neoformans wild-type and cwc1 mutant strains. Using the mutant as the recipient strains, the overexpression effect of the truncated gene can be purely scored and the importance of deleted region can thus be determined. When using the wild-type strain, the phenotype may imply if the truncated protein still retains the interaction ability. If deleted the region important for protein interaction, the phenotype of the transformant will be similar to the wild-type strain since the functional complex can be formed by the endogenous wild-type proteins. The transformants with elevated levels of the truncated CWC1 transcripts were verified by Northern blot analysis (data not shown) and subjected to phenotypic characterization. If the LOV domain is essential for the function of CwC1, overexpression of the CWC1 gene lacking the LOV domain should fail to inhibit sexual filamentation upon light activation. As expected, overexpression of the $C W C 1_{(568-680 \triangle)}$ gene in the $c w c 1$ mutant background (Fig. 1Bf) produced strains that were insensitive to light and filament production in these transformants were similar to the original cwc1 mutant (Fig. 1Bb). Overexpression in the wild-type also yielded light insensitive transformants, indicating that overexpression of the $C W C 1_{(568-680 \triangle}$ gene had a dominant negative effect on light regulation (Fig. 1Be). In contrast, filament production in the wild-type cross was inhibited by light (Fig. 1Ba) and overexpression of the wild-type CWC1 gene in the wild-type strain (Fig. 1Bc) and cwc1 mutant (Fig. 1Bd) both completely inhibited filamentation under light conditions.

The importance of the PAS domains in Cwc1 was also confirmed by overexpression of the $C W C 1_{(767-929 \triangle)}$ gene that lacked the PAS domains (Fig. 1A). The $c w c 1$ mutant overexpressing $C W C 1_{(767-929 \triangle)}$ resembled the $c w c 1$ mutant (Fig. $1 \mathrm{Bh}$ and $\mathrm{Bb}$ ); whereas, overexpression of the $C W C 1_{(767-929 \triangle)}$ construct in the wild-type background was similar to the wild-type strain (Fig. $1 \mathrm{Bg}$ and $\mathrm{Ba}$ ). Our results demonstrated that the LOV and PAS domains are essential for the function of C. neoformans Cwc1 protein.

\subsection{The PAS domain and GATA-type zinc finger motif of the Cwc2 protein are important for the light regulation in $C$. neoformans}

Based on the predicted structural feature, C. neoformans Cwc2 protein contains a putative PAS domain and GATA-type zinc finger DNA-binding motif (Lu et al., 2005). The PAS domain is involved in protein-protein interaction and the zinc finger DNA-binding motif is a feature of transcription factor. To confirm their importance, we similarly generated truncated versions of the $C W C 2$ gene under control of the GPD1 promoter and no sequence change during amplification was also confirmed (Fig. 2A). The expression level of truncated transcripts was also verified by Northern blot analysis (data not shown). Overexpression of the $C W C 2_{(72-140 \triangle}$ ) gene, which lacked the PAS domain, in the wild-type background (Fig. 2Be) displayed the same mating phenotype as the wild-type strain (Fig. 2Ba), while in the cwc2 mutant background (Fig. 2Bf) the strain displayed the phenotype as the $c w c 2$ mutant (Fig. 2Bb). Furthermore, overexpression of the $C W C 2_{(349-373 \triangle)}$ gene, which was deleted for the zinc finger motif, in the $c w c 2$ mutant background (Fig. 2Bh) produced filaments similar to the $c w c 2$ mutant (Fig. 2Bb). In the wild-type background (Fig. 2Bg), the level of filamentation was intermediate between the wild-type (Fig. 2Ba) and $c w c 2$ mutant strains (Fig. 2Bb), suggesting a complex poisoning or semi-dominant negative effect by the mutation version of Cwc2. These results demonstrate that PAS domain and GATA-type zinc finger motif of the Cwc2 protein are also crucial for blue light responses in $C$. neoformans.

A
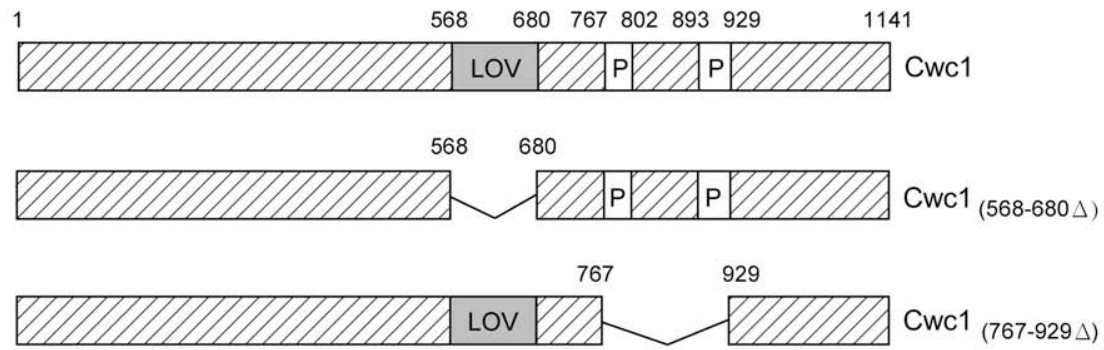

B

MATa WT

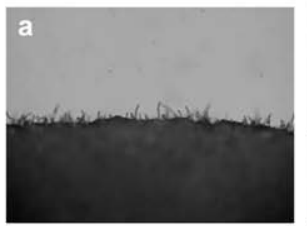

MATa cwc1

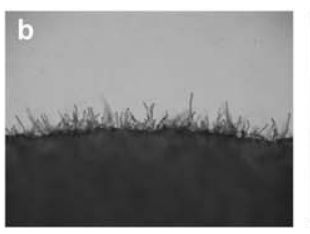

MATa pGPD1::CWC1

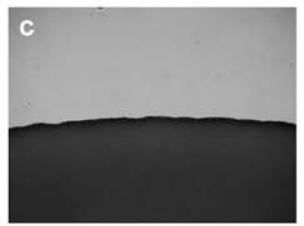

MATa cwc1

+ pGPD1::CWC1

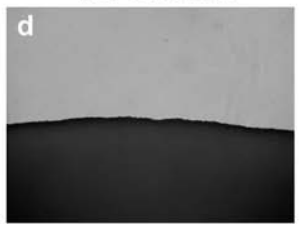

MATa pGPD1::CWC1(568-680 $\triangle$

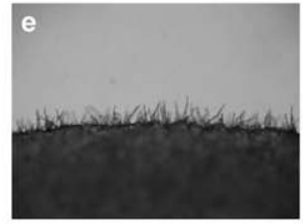

MATa cWc1
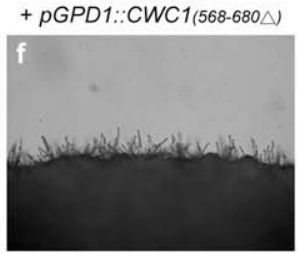

MATa pGPD1::CWC1(767-929

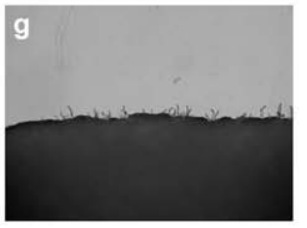

MATa cWc1

+ pGPD1::CWC1(767-929N

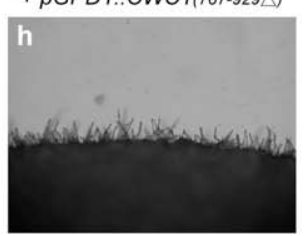

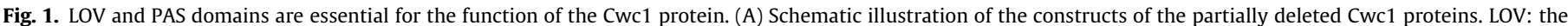

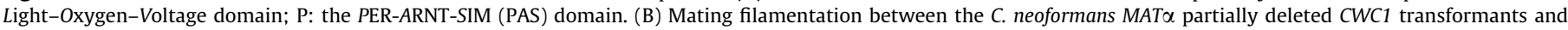
MATa wild-type strain (JEC20) on V8 agar medium at $26^{\circ} \mathrm{C}$ for $18 \mathrm{~h}$ under light condition. The edges of mating mixtures were photographed at a magnification of $100 \times$. 


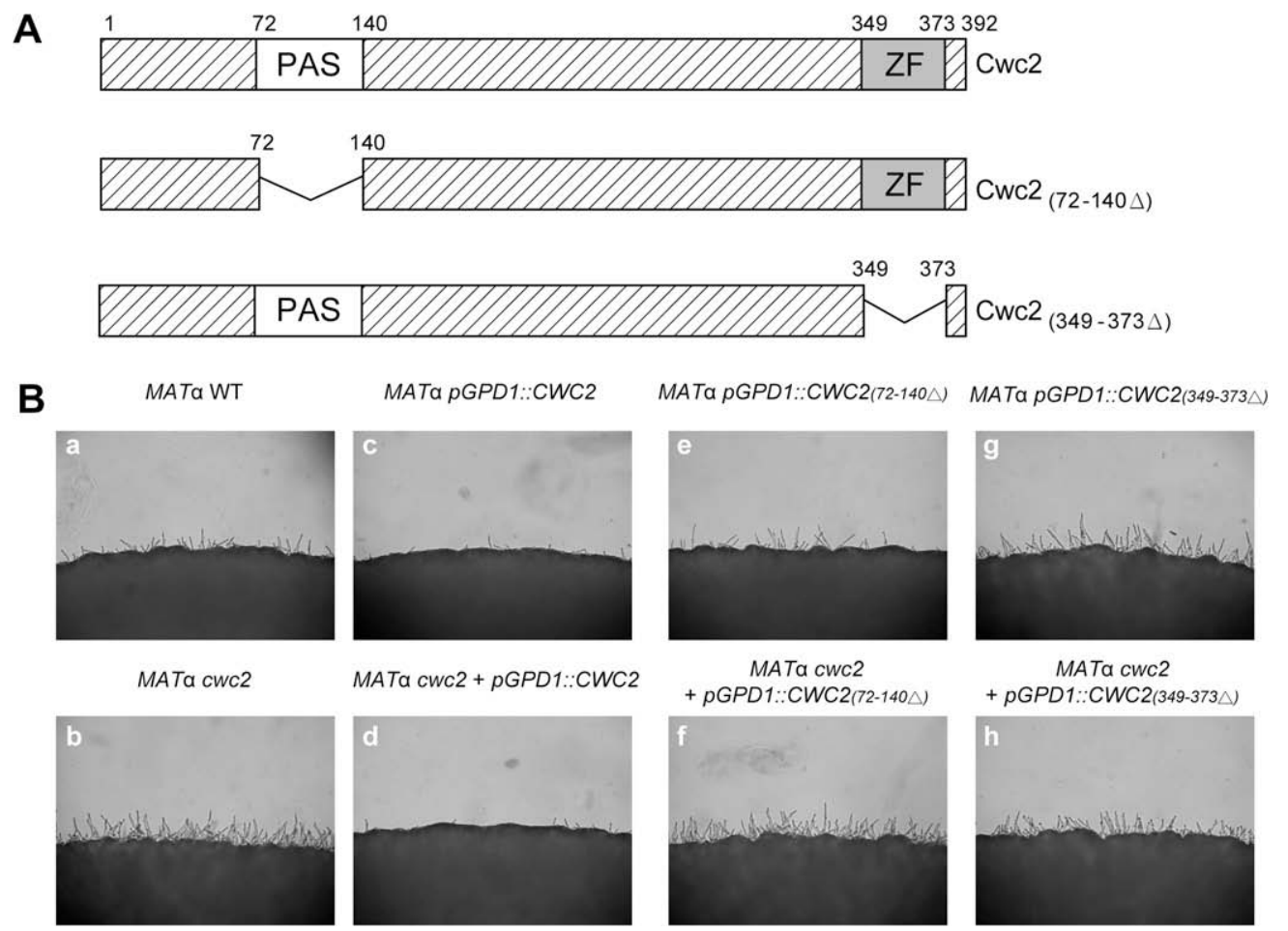

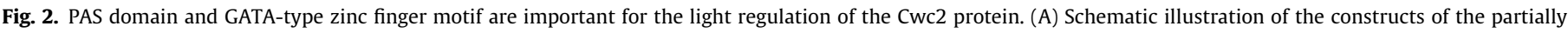

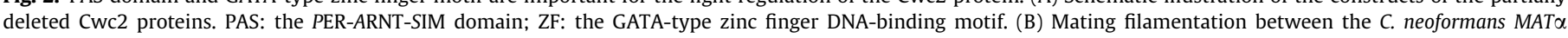

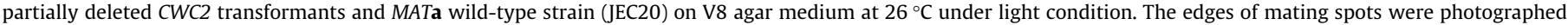
after $18 \mathrm{~h}$ incubation at a magnification of $100 \times$.

\subsection{Cryptococcus neoformans $C w c 1$ and $C w c 2$ proteins function interdependently to regulate light response}

Based on the conservation of the PAS domains in the Cwc1 and Cwc2 proteins and also similar phenotypes observed in the $C W C 1$ and $C W C 2$ gene deletion or overexpression strains, $C$. neoformans Cwc1 and Cwc2 may form a complex to regulate downstream related genes. To test their relationship genetically, we created the CWC2 overexpression strains in the $c w C 1$ mutant background and also the CWC1 overexpression strains in the $c w c 2$ mutant background. The elevated expression of the $C W C 2$ and $C W C 1$ transcripts in these strains was verified by Northern blot analysis (data not shown). The confirmed strains were examined for their mating phenotypes under light illumination. As predicted, overexpression in the mutant background in either case (Fig. 3G and $\mathrm{H}$ ) failed to inhibit sexual filamentation upon light induction, and their phenotypes all resembled to the $c w c 1$ and $c w c 2$ single or double mutants (Fig. 3B-D). These findings demonstrate that C. neoformans Cwc1 and $\mathrm{Cwc} 2$ proteins function interdependently to regulate photoresponses, which is consistent with a biochemical study shown by the yeast two-hybrid assay that the two proteins physically interact (Idnurm and Heitman, 2005).

\subsection{Dissecting the C. neoformans blue light-inhibited filamentation pathway by screening for suppressor mutants}

In order to identify genes interacting with or acting downstream of the Cwc complex in the blue light-inhibited filamenta-
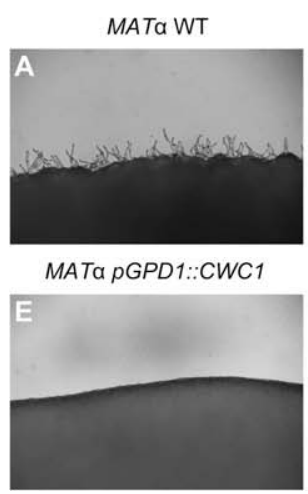

MATa $c W c 1$

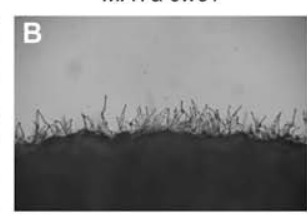

MATa $p G P D 1:: C W C 2$

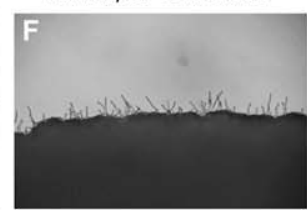

MATa $c w c 2$

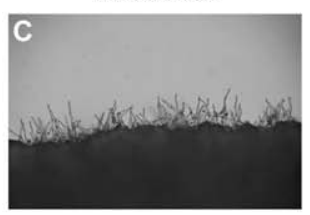

MATa $c W C 1+p G P D 1:: C W C 2$

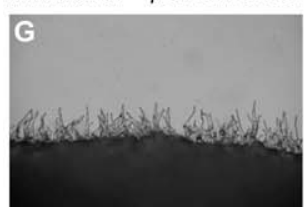

MATa $c w c 1$ cwc2

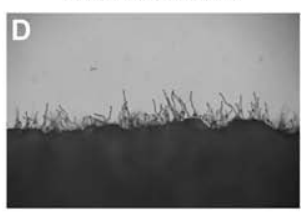

$M A T \alpha c W c 2+p G P D 1:: C W C 1$

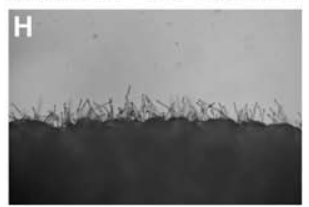

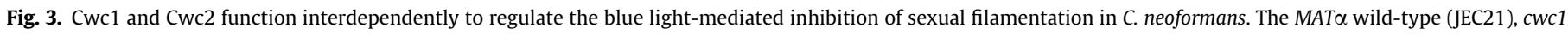

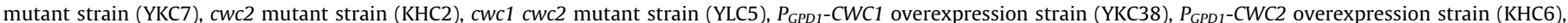

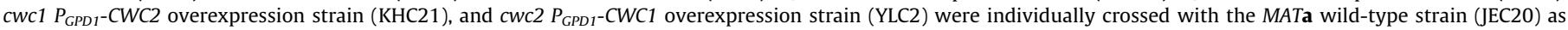
indicated. Mating was conducted on V8 agar medium at $26^{\circ} \mathrm{C}$ under light condition, and photos were taken after $20 \mathrm{~h}$ incubation at $100 \times$ magnification. 
tion pathway in C. neoformans, a screening mediated by Agrobacterium insertional mutagenesis was conducted to isolate T-DNA mutants which suppressed the light-dependent inhibition of mating filamentation phenotype in the CWC1 overexpression strain. Overexpression of the CWC1 gene was proposed to up-regulate genes with negative roles in the pathway and lead to complete inhibition of sexual filamentation upon light induction (Lu et al., 2005). Therefore, identification of mutants restoring the filamentation will reveal $C$. neoformans genes important for negative regulation of sexual filamentation in response to light. The filamentation phenotype we screened is a consequence of cell fusion between two haploid parents. Since one wild-type copy derived from the MATa wild-type parent is present in the dikaryotic hyphae, our screen will not detect the mutation with recessive nature.

To conduct Agrobacterium insertional mutagenesis, the CWC1 overexpression strain was co-incubated with $A$. tumefaciens cells containing the NAT delivery plasmid for 2 days and spread onto selection media containing nourseothricin and cefotaxime. After single colony isolation, 10,341 nourseothricin-resistant transformants were obtained and mating assays were conducted with the MATa wild-type strain. Of these 10,341 strains, 134 transformants which restored sexual filamentation under light illumina- tion were verified after repeating three round of mating assays. The ratio of filament-producing suppressors in our screen was about $1.3 \%$. We classified suppressor strains into five categories according to the amount of filamentation produced in the mating assay under light irradiation. Mutants producing a filamentation level similar to the wild-type were designated as level 5 and AZ5 mutant was an example (Fig. 4C). Intermediate levels of filamentation were classified from level 4 to 1 (Fig. 4D-G). We obtained six mutants classified as level 5, 16 mutants as level 4, and 28, 30, and 54 mutants as level 3, 2, and 1, respectively.

The copy number and fate of transforming T-DNA in the filamentous suppressors were further determined by Southern analysis with NAT selectable marker. So far, the copy number of T-DNA integration has been determined among 66 transformants, of which 47 were one-copy, 15 were 2 copies, and 4 were 3 copies. T-DNA integration sites of selected strains have been recovered by inverse PCR. Products of expected sizes were sequenced, and blast analyses were conducted to identify the disrupted genes. Currently, 17 T-DNA integrated sites have been identified and their filamentation phenotypes associated with mating and monokaryotic fruiting are listed in Table 3. Since background mutation may occur in the transformants along the procedure, the linkage of T-DNA

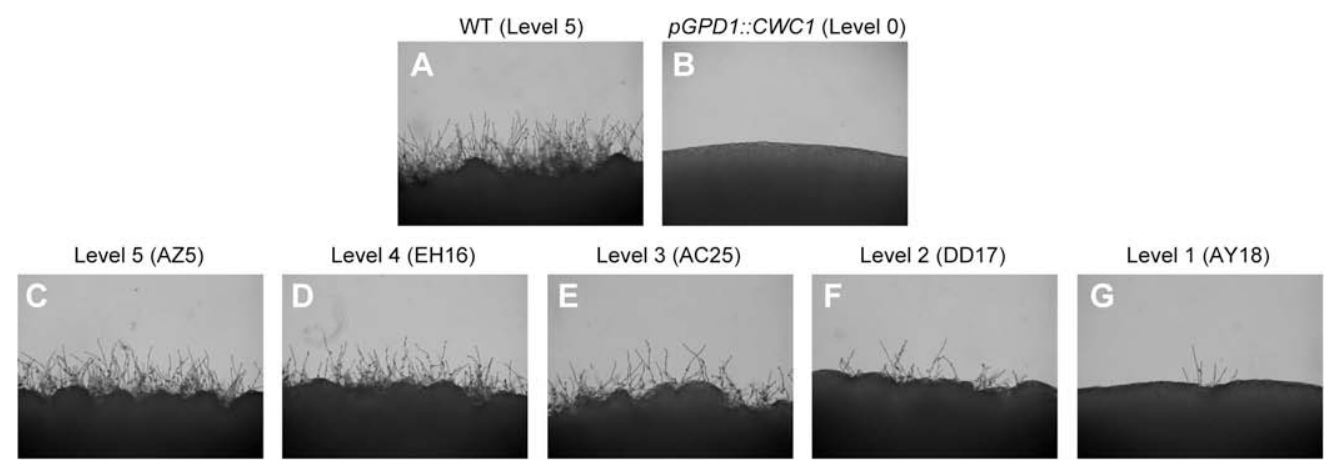

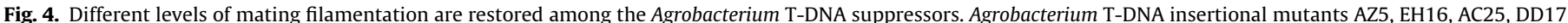

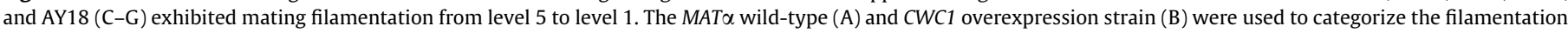

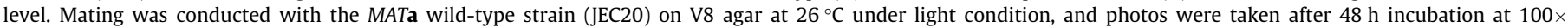
magnification.

Table 3

ATMT transformants in this study.

\begin{tabular}{|c|c|c|c|c|c|}
\hline Strains & Related gene & Locus No. & Mating $^{\mathrm{a}}$ & Fruiting ${ }^{\mathrm{b}}$ & Linkage $^{c}$ \\
\hline AZ5 & CWC2 gene & CNE01220 & 5 & 0 & NA \\
\hline AY18 & General RNA polymerase II transcription factor & CNA04230 & 1 & 5 & + \\
\hline AC25 & DNA mismatch repair protein Pms1 & CNC02500 & 3 & 0 & NA \\
\hline AS31 & Histone-lysine $N$-methyltransferase & CNG03810 & 2 & 0 & + \\
\hline BU26 & Structural maintenance of chromosomes (SMC) protein & CND06390 & 3 & 0 & NA \\
\hline CZ31 & UXS1 gene & CNG02560 & 1 & 0 & NA \\
\hline DD17 & Nicotinamide mononucleotide permase & CNA05810 & 2 & 0 & NA \\
\hline DI6 & Hypothetical protein & CNE02520 & 1 & 0 & NA \\
\hline DJ22 & Cdk-related kinase 1: CRK1 & CNM01920 & 1 & 0 & + \\
\hline DT21 & Hypothetical protein & CNF04850 & $3-4$ & 0 & NA \\
\hline EG30 & Bud site selection protein & CNL04250 & $4-5$ & 0 & + \\
\hline EH16 & Amino acid transporter & CNB04970 & 4 & 0 & NA \\
\hline ET21 & Conserved hypothetical protein & CNC02650 & 1 & 0 & NA \\
\hline GC17 & Intergene (between two hypothetical proteins) & $\begin{array}{l}\text { CNL04560 } \\
\text { CNL04570 }\end{array}$ & 4 & - & NA \\
\hline GJ5 & Vacuole protein & CNI02790 & 1 & - & NA \\
\hline GK17 & Intergene (between pre-mRNA splicing factor: PRP1 and hypothetical protein) & $\begin{array}{l}\text { CNA00700 } \\
\text { CNA00710 }\end{array}$ & 2 & - & NA \\
\hline NO7 & Expressed protein & CNI03950 & 1 & 0 & NA \\
\hline
\end{tabular}

${ }^{\text {a }}$ Mating assay was conducted on V8 agar at $26{ }^{\circ} \mathrm{C}$ under light condition for $48 \mathrm{~h}$. No filamentation designated as level 0 ; filamentation level of the AZ5 and JEC21 strain designated as level 5 .

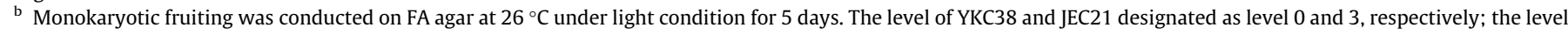
of AY18 designated as level 5.

${ }^{c}+$, the linkage of T-DNA insertion and observed phenotype was established by complementation or progeny segregation analyses. NA, not available. 
insertion and observed phenotype established by complementation experiments in few strains was also indicated in Table 3.

\subsection{T-DNA integration in the AZ5 strain, which restored the highest} filamentous phenotype, was found at the CWC2 locus

To find the $C$. neoformans genes important for the photoresponse, we chose suppressor mutants from level 5 as initial targets for characterization. Their high and similar filamentation level in the light and dark indicates they may be "blind" and the genes disrupted in these strains might function with Cwc complex or at the early steps of the photoregulatory pathway. One of these mutant strains, AZ5, suppressed the light-dependent inhibition of filamentation by the CWC1 overexpression and restored abundant filamentation under light and dark conditions (Figs. 4C and 5A; data not shown). One copy of T-DNA was found by Southern blot analysis to integrate in the AZ5 strain (Fig. 5B). Sequencing the recovered PCR product revealed that T-DNA with the NAT selectable marker was inserted $67 \mathrm{bp}$ upstream of the CWC2 start codon (Fig. 5C and $\mathrm{D}$ ). Integration at the $C W C 2$ locus was further verified by
Southern hybridization with the $C W C 2$ probe (Fig. 5B). Furthermore, based on Northern analysis, we confirmed that the CWC2 gene was not expressed in the AZ5 strain due to the T-DNA insertion (data not shown). The mating phenotype of AZ5 was similar to that of the $C W C 1$ gene overexpression in the $c w c 2$ mutant background (Fig. 5A). This finding confirmed that Cwc2 is an important component of the blue light-inhibited filamentation pathway in $C$. neoformans and also validated our screening approach.

3.6. AY18 suppressor restored limited amount of mating filaments but showed dramatic derepression of monokaryotic filamentation

Among the 134 filamentous suppressors identified by mating assay, 17 strains have been subjected to monokaryotic filamentation assay. Two suppressor mutants, AY18 and DN16, were found capable of producing monokaryotic fruiting filaments in the CWC1 overexpression background. The mating filamentation levels for AY18 and DN16 were categorized as level 1 and 3, respectively (Fig. 4 and Table 3; data not shown). The extent of monokaryotic filamentation was examined in few mutants and also briefly cate-
A

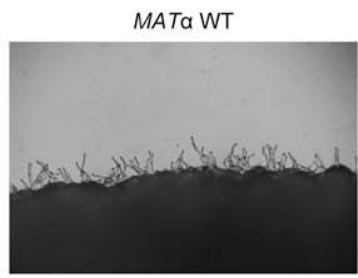

MATa cwc2

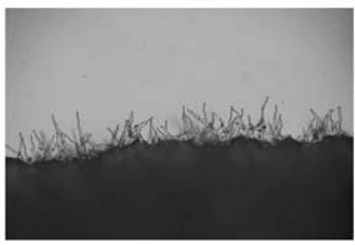

B

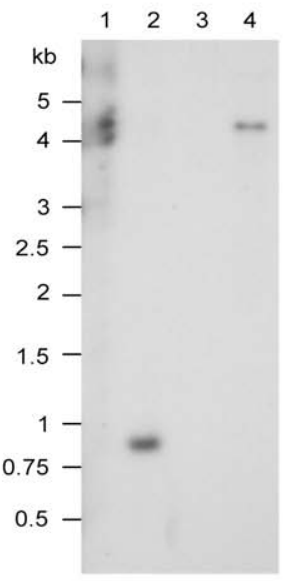

MATa pGPD1::CWC1

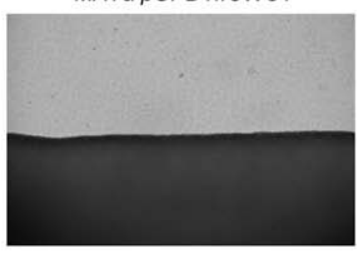

MATa $C W C 2+p G P D 1:: C W C 1$

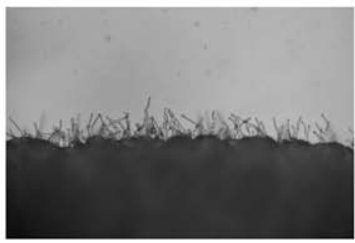

MATa cwc1

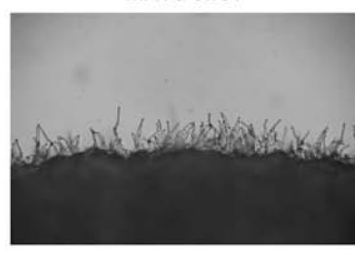

AZ5

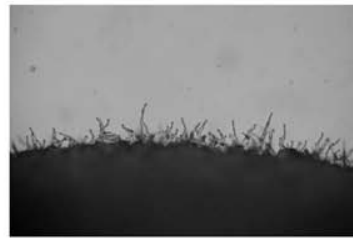

C
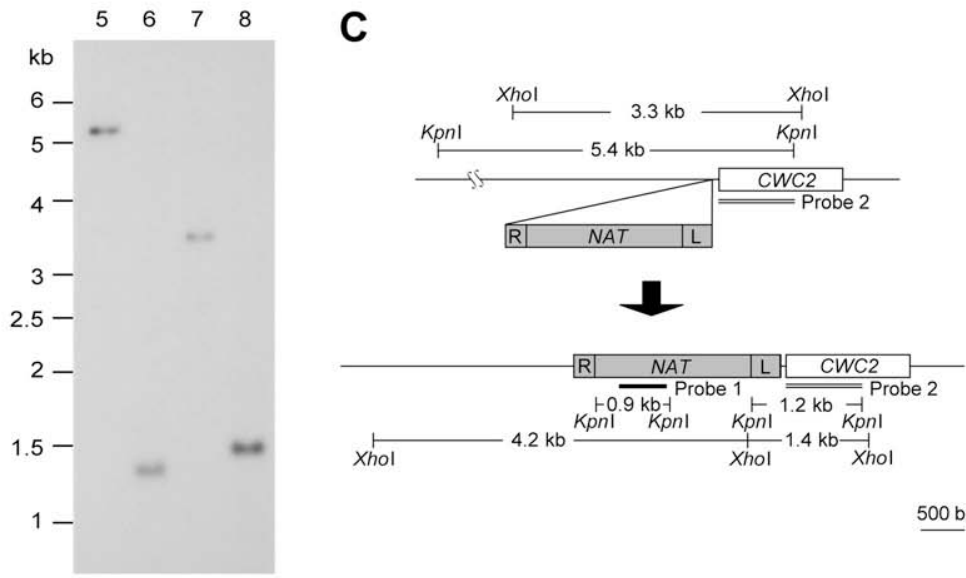

$500 \mathrm{bp}$

D

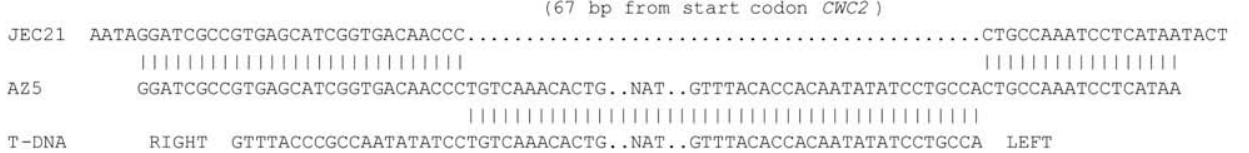

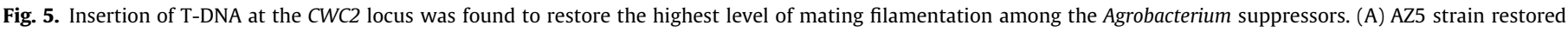

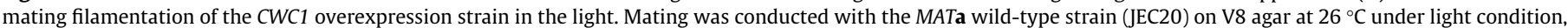

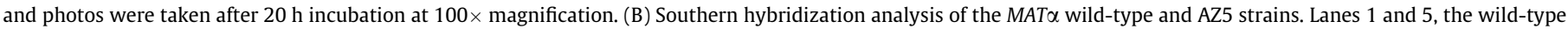

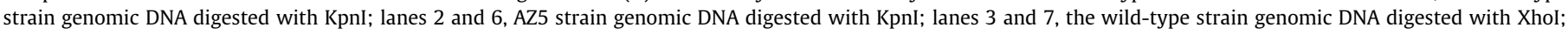

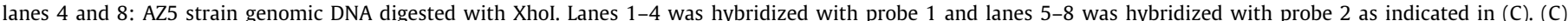

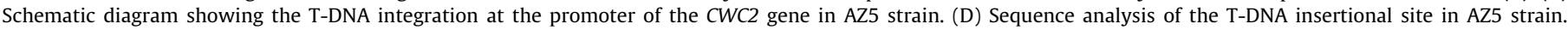
Sequences of the wild-type JEC21, AZ5 strain, and the right and left borders of T-DNA were aligned. 
gorized. For the CWC1 overexpression strain and most of the suppressor mutants listed in Table 3, no filamentation was observed under light illumination (level 0 ). The filamentation level normally seen in the MAT $\alpha$ wild-type strain was designated as level 3 and DN16 was similar to the MAT $\alpha$ wild-type strain. AY18 interestingly showed more dramatic production of monokaryotic filaments when compared to the MAT $\alpha$ wild-type strain and was categorized as level 5 (Fig. 6A). Due to its interesting phenotype in the monokaryotic filamentation, AY18 strain was selected for further characterization.

\subsection{Progeny segregation analysis of the AY18 suppressor}

To determine the genetic linkage between the AY18 phenotypes and the NAT integration, we first verified the copy number of TDNA integration in the AY18 strain. The results of Southern hybridization indicated that 3 copies of T-DNA were present in the AY18 genome (Fig. 6B). Segregation analysis was then conducted on the mating progeny of the AY18 and MATa wild-type strain. To prevent parental yeast cell contamination, we developed a technique which restricts the parental yeast cells by making a gapped circular area around the mating colony and allows isolating the basidiospores from the mating filaments crossing the circular gap. Progeny were selected on nourseothricin containing YPD plates. Among the 96 resistant strains, 29 strains were uracil auxotrophic and not subjected to further analyses (data not shown). Using the STE12 $\alpha$ / STE12a genes as the PCR targets for mating type determination (Fig. 7Aa and Ab), 28 MAT $\alpha$ and 16 MATa strains were confirmed, suggesting that the MAT locus represented an independently segregating genetic marker. Among these 44 strains, 29 strains contained the intact $P_{G P D 1}-C W C 1$ expression cassette (Fig. 7Ac), and
43 strains retained the T-DNA integration pattern at the SSN8 locus as revealed by PCR analyses (Fig. 7Ad; described in Section 3.8). Nine selected strains were further verified by Southern hybridization and all three original copies of inserted T-DNA were found to be present in these strains (Fig. 7B). Based on the genotypic analyses among the nourseothricin-resistant progeny, we conclude that the integration of three copies of T-DNA was linked in the AY18 strain and the integration site responsible for the AY18 phenotypes remained unclear.

\subsection{T-DNA integration at a mediator gene, SSN8, resulted in the restoration of mating filamentation and dramatic derepression of monokaryotic filamentation in the AY18 suppressor}

Since all three T-DNAs appeared to be tightly linked in the AY18 strain, we failed to separate the insertion sites by sexual recombination. However, due to its interesting phenotypes, we decided to define all three integration sites. Digestion with HindIII yielded 2.5, 8.2 , and $11 \mathrm{~kb}$ fragments using the NAT gene as the probe (Fig. 6B). Inverse PCR was first attempted to recover the insertion site around the $2.5 \mathrm{~kb}$ fragment and a product of the expected size from one flanking region was successfully obtained and sequenced (Fig. 6C). Blast searches against the GenBank and C. neoformans genome databases revealed that T-DNA was inserted at the N-terminus of an SSN8 homologue of Saccharomyces cerevisiae (Fig. 6C and D). S. cerevisiae SSN8 gene is a cyclin-like component of the RNA polymerase II and is involved in a wide array of cellular processes.

To determine if the phenotypes of AY18 was due to the disruption of the SSN8 gene, we made the SSN8 gene deletion in the CWC1 overexpression background. The SSN8 gene was replaced with the
A

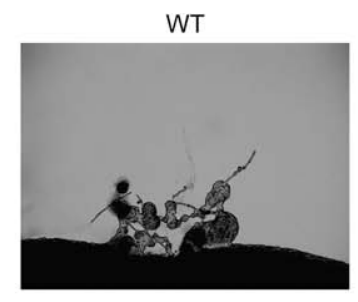

B

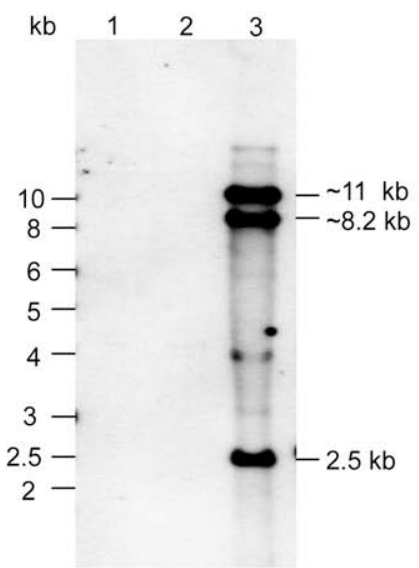

pGPD1::CWC1

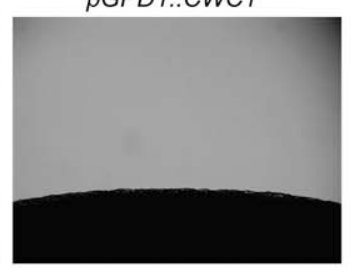

C
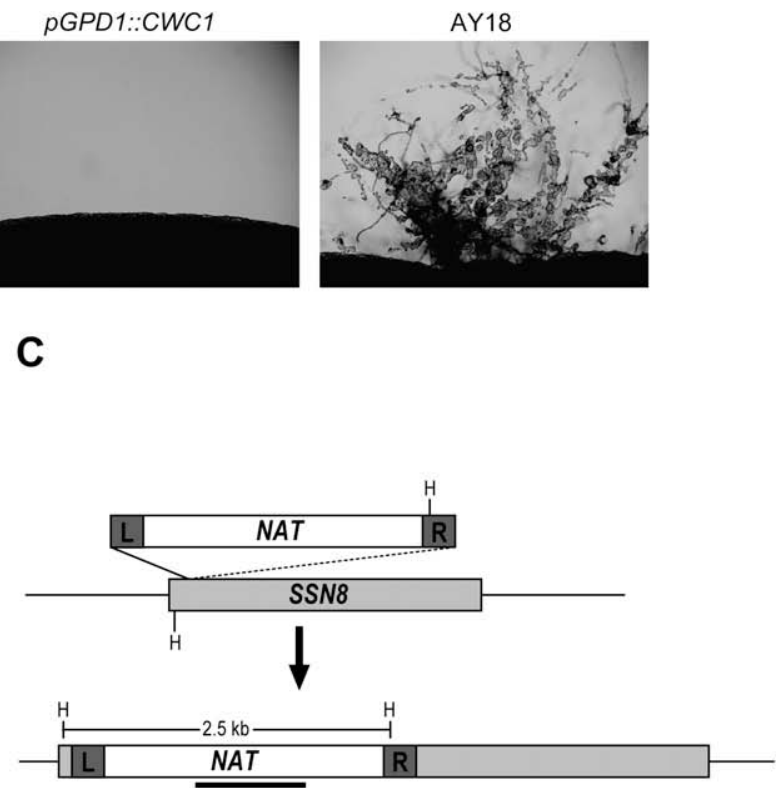

D

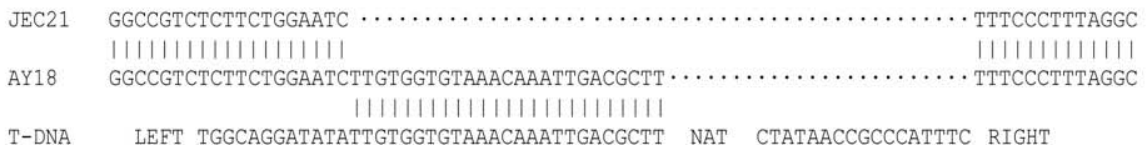

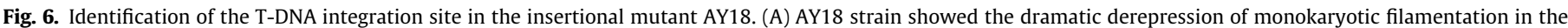

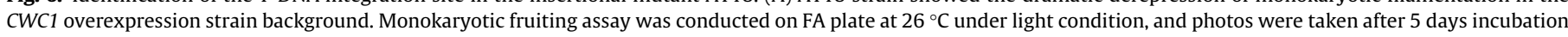

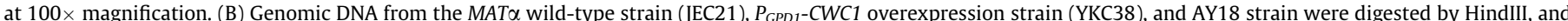

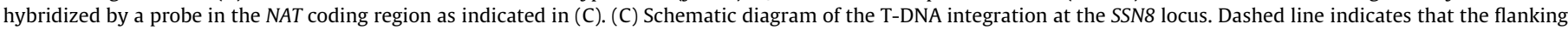
insertion site has not yet been verified. (D) Sequences of the wild-type JEC21, AY18 strain, and T-DNA left border were aligned. 


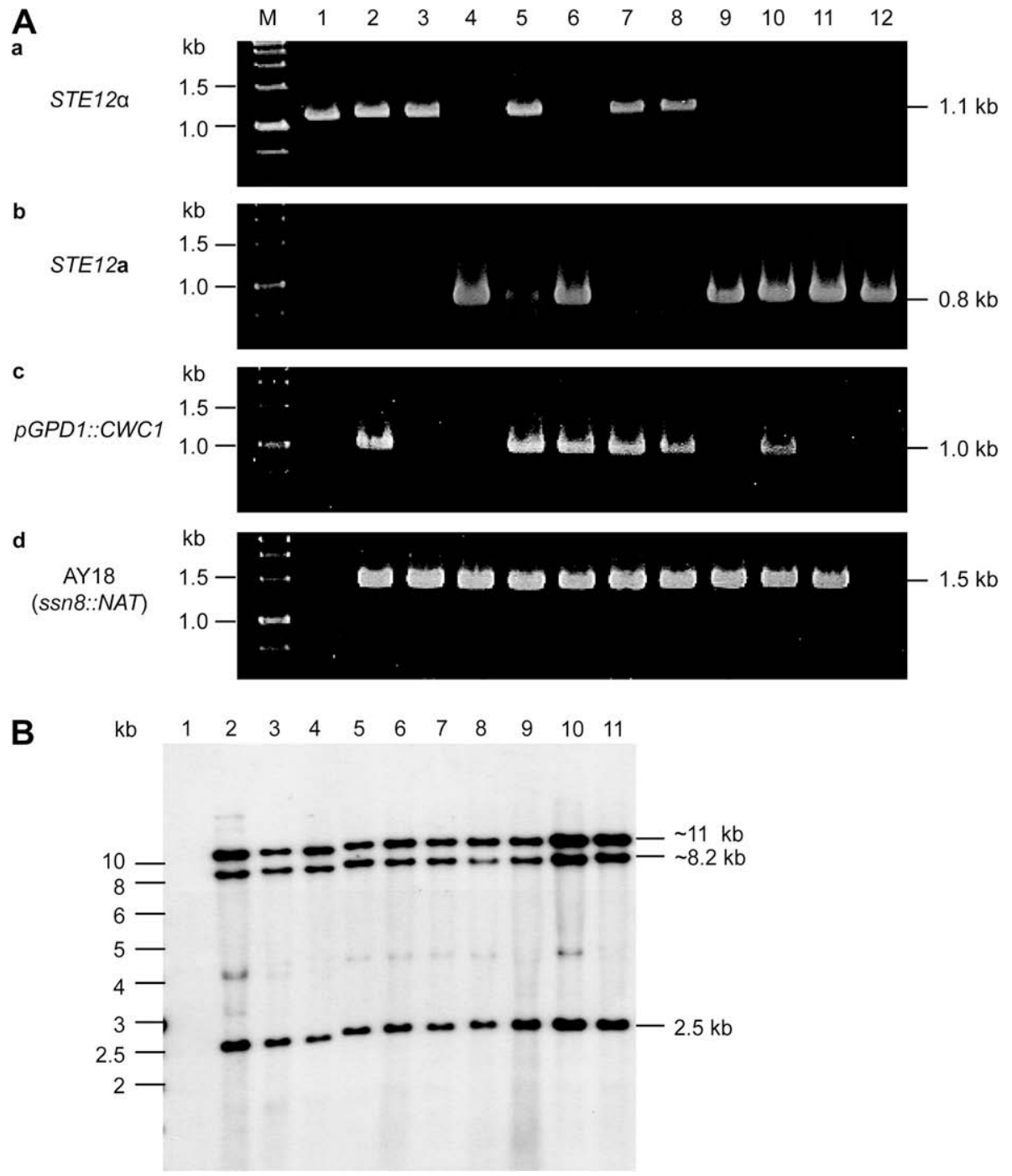

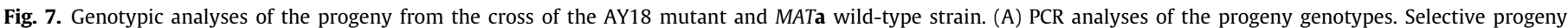

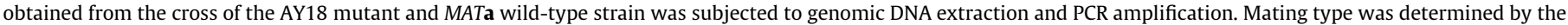

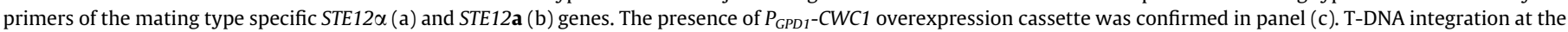

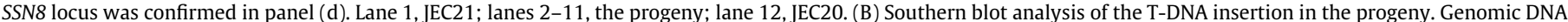
was digested by HindIII, and hybridized by a probe in the NAT coding region. Lane 1, JEC21; lanes 2-11, the progeny.

NAT selectable marker and transformed into the CWC1 overexpression strain YKC38 (Fig. 8A). The ssn8 gene deletion mutant was verified by Southern hybridization with different restriction enzymes (Fig. 8B) and subjected to phenotypic comparisons with the AY18 mutant. We found that deletion of the SSN8 gene in the CWC1 overexpression strain reproduced mating filamentation and dramatic derepression of monokaryotic filamentation seen in the AY18 strain. Furthermore, complementation by the wild-type copy SSN8 gene in the AY18 mutant and SSN8-deleted CWC1 overexpression strain restored these filamentation phenotypes back to the CWC1 overexpression strain (data not shown). Therefore, we conclude that T-DNA disruption at the SSN8 gene was responsible for the phenotypes of the AY18 strain.

\section{Discussion}

Light displays profound effects on fungal biology. Evidences indicate that evolutionally conserved white collar proteins function as key blue light regulators in fungi across diverse taxonomical groups (Corrochano, 2007; Herrera-Estrella and Horwitz, 2007; Purschwitz et al., 2006). Structural comparisons of the WC-1 and
WC-2 orthologues from two major fungal phyla, Ascomycete and Basidiomycete, suggest that these proteins might operate in a similar but not identical way in executing their regulatory mechanisms (Corrochano, 2007; Idnurm and Heitman, 2005; Lu et al., 2005).

To understand how Cwc1 and Cwc2 regulate light responses, we characterized their putative protein domains with conserved homology by deletion studies. C. neoformans Cwc1 was predicted to contain the chromophore-binding LOV domain and protein interacting PAS domains (Lu et al., 2005). Overexpression of the LOV domain-deleted $C W C 1_{(568-680 \triangle)}$ in the $c w c 1$ mutant background maintained the light insensitivity and displayed phenotypes similar to the original $c w c 1$ mutant (Fig. $1 \mathrm{Bf}$ and $\mathrm{Bb}$ ). C. neoformans cwc1 mutant containing the PAS domains-deleted $C W C 1_{(767-929 \triangle)}$ gene also exhibited phenotypes similar to the cwc1 mutant (Fig. 1Bh and Bb). Since deletion of a segment of protein region may affect the general protein structure, the importance of specific region may not be truly revealed. However, our preliminary results found that deletion of a 148 amino acids region at the N-terminal part of Cwc1 showed no impact on the Cwc1 function (data not shown). Therefore, based on our results, we con- 

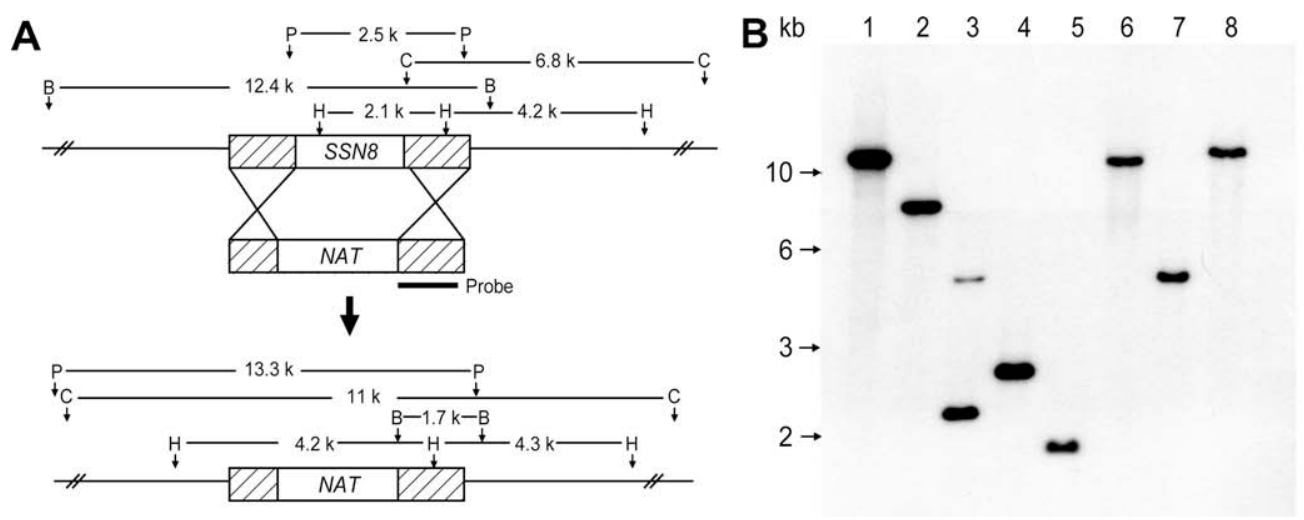

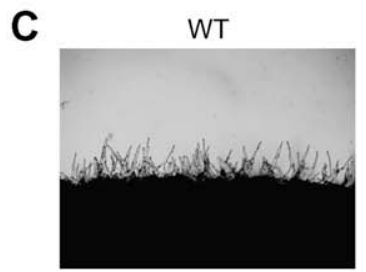

WT

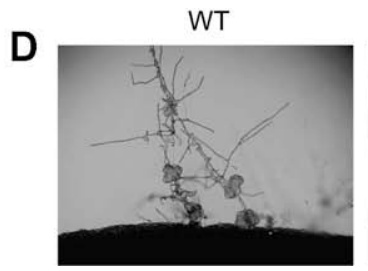

pGPD1::CWC1

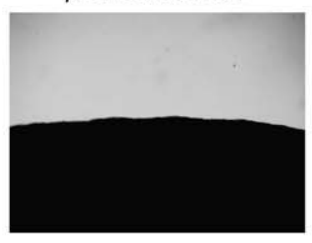

pGPD1::CWC1

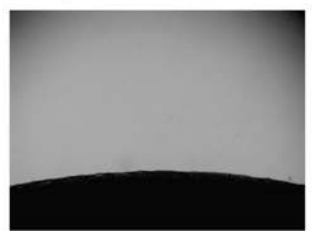

AY18

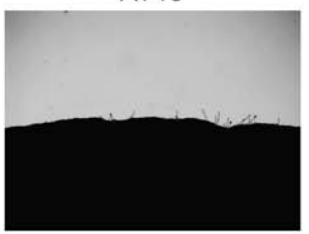

AY18

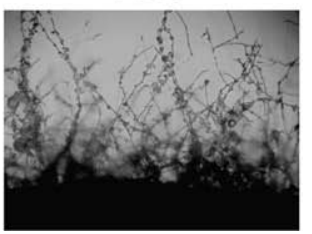

pGPD1::CWC1 ssn8

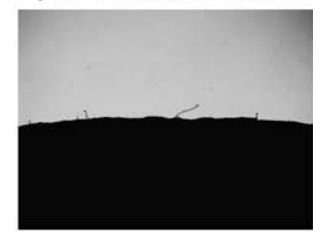

pGPD1::CWC1 ssn8

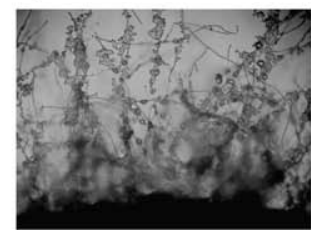

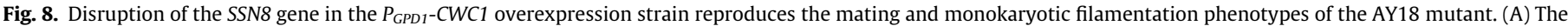

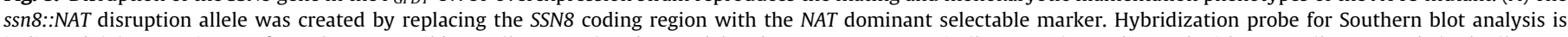

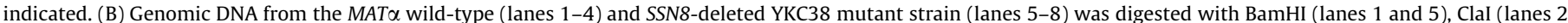

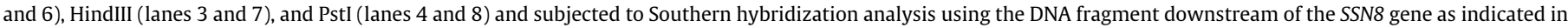

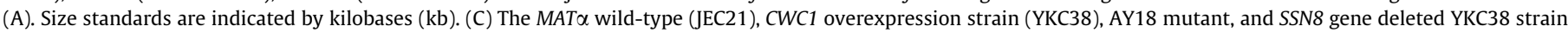

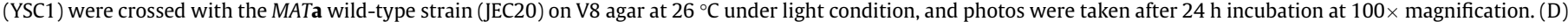

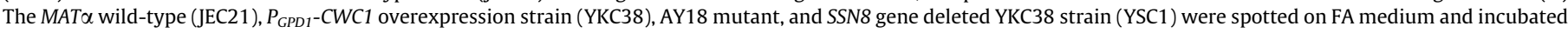
at $26^{\circ} \mathrm{C}$ under light condition. Photos were taken after 5 days incubation at $100 \times$ magnification.

clude that LOV and PAS domain play crucial role for the function of Cwc1.

Overexpression of the CWC1-truncated constructs in the wildtype strain showed interesting phenotypes. The wild-type strain containing the $C W C 1_{(568-680 \triangle)}$ overexpression construct showed phenotype similar to the $c w c 1$ mutant (Fig. $1 \mathrm{Be}$ and $\mathrm{Bb}$ ). This result indicates that the non-functional LOV-deleted Cwc1 proteins had a complex poisoning effect and displayed a dominant negative effect over the wild-type Cwc1 proteins. The non-functional LOV-deleted Cwc1 proteins could compete with the wild-type Cwc1 proteins for binding to $\mathrm{Cwc} 2$, and proper association of the normal Cwc proteins was thus blocked. This complex poisoning phenomenon was not seen in the PAS domains-deleted $\left.\mathrm{CwC1}_{(767-929 \triangle}\right)$ wild-type strain and its filamentation level was slightly less than the wildtype strain (Fig. 1Bg). The phenotype suggests that the endogenous wild-type Cwc1 proteins can still interact normally with Cwc2 to inhibit filamentation, and the PAS domains-deleted Cwc1 proteins failed to compete for the Cwc2 proteins in vivo. The cause for slight reduction of the filamentation level in this strain is unknown. Whether alteration of the endogenous wild-type CWC1 transcript level contributes to the phenotype requires further investigation.

Overexpression of the intact $C W C 2$ gene also inhibits sexual filamentation under light condition; however, the inhibition is not as dramatic as the CWC1 overexpression strain (Lu et al., 2005). In our deletion studies, overexpression of the PAS domain-deleted CWC2 $(72-140 \triangle)$ in the $c w c 2$ mutant showed the phenotype similar to the cwc2 mutant (Fig. 2Bf and $\mathrm{Bb}$ ), and the same construct in the wild-type strain displayed the phenotype similar to the wild-type strain (Fig. 2Be and Ba). These phenotypes suggest that the PAS domain-deleted Cwc2 proteins are not functional and also fail to interfere with the interaction between the wild-type Cwc1 and Cwc2 proteins. Therefore, the importance of PAS domain in the Cwc2 protein is confirmed.

Surprisingly, overexpression of the zinc finger motif-deleted $C W C 2_{(349-373 \triangle)}$ construct in the $c w c 2$ mutant background produced filaments similar but not to the same level as the cwc2 mutant (Fig. 2Bh). This observation was repeatedly seen among different transformants and also verified in different experiments. In addition, overexpression of the same construct in the wild-type strain also showed a filamentation phenotype intermediate between the $c w c 2$ mutant and the wild-type strain (Fig. $2 \mathrm{Bg}$ ). One possible explanation is that the truncated $\mathrm{CwC}_{(349-373 \triangle)}$ protein may still retain partial DNA-binding activity due to the flanking or other regions of the protein. Recent deletion experiment found that the last 19 amino acids of Cwc2, also located next to the zinc finger motif, were also crucial for the Cwc2 function (data not shown).

Previous studies by our group and others demonstrated that blue light inhibits sexual filamentation via the Cwc/Bwc complex in C. neoformans (Idnurm and Heitman, 2005; Lu et al., 2005). This negative effect was further supported by our overexpression study, in which overexpression of the $C W C 1$ or $C W C 2$ gene enhances the inhibition phenotype upon light activation (Lu et al., 2005). Based on these results, we have proposed two models to depict the possible regulatory network(s) in $C$. neoformans. In model 1 , the light- 
activated Cwc complex binds directly to the promoters and negatively regulates gene involved in the filamentation of mating process. In model 2, a repressor functions intermediately between the Cwc complex and downstream genes. In response to light, Cwc complex activates the transcription of the repressor and then the repressor subsequently inhibits genes involved in the mating filamentation. To further dissect this pathway, we have conducted Agrobacterium random mutagenesis screen in the CWC1 overexpression strain. Our screen was designed to identify mutants suppressing the CWC1 overexpression effect. The CWC1 overexpression strain shows complete inhibition of sexual filamentation upon light irradiation and its dominant and tight phenotype allowed us to easily score the T-DNA mutants restoring the filamentous growth. However, only mutations with the dominant effect will be revealed in our mating screening. Agrobacteriummediated transformation has been proven a powerful tool for functional genomics in fungi (de Groot et al., 1998; Michielse et al., 2005). In C. neoformans, Agrobacterium-mediated insertional mutagenesis displayed advantageous features including random integration, high transformation efficiency and improved stability over other transformation systems (Idnurm et al., 2004) and has also been successfully used to identify genes with novel functions in combination with available genomic sequence resources (Walton et al., 2005).

In our screen, we expected that genes mutated might be part of the Cwc complex or function as regulators in direct or indirect conjunction with the Cwc complex for light perception and transduction or light-regulated filamentation pathway. From our screen, we obtained a significant number of suppressor mutants. The number is not surprising, since that a large number of QTLs (quantitative trait loci) affecting filamentation has been demonstrated (Lin et al., 2006). Among the filamentous suppressors, most of them restored limited amount of filaments; however, over twenty suppressors produced mating filaments similar to, or even more than, the wild-type strain. Comparison of the filamentation phenotypes in the light and dark might reflect the roles or steps of these genes in controlling the processes. One type of the mutants we expected is the "blind" mutants, which should produce abundant and similar level of filaments in the light and dark. Although T-DNA has not been found to disrupt the CWC1 overexpression, we did identify mutants affecting the $C W C 2$ gene. In addition to AZ5, we recently found three other mutants in level 5 , in which T-DNAs were inserted at the promoter or coding region of the $C W C 2$ gene (results not shown). The light regulatory role of Cwc2/Bwc2 in $C$. neoformans was previously confirmed (Idnurm and Heitman, 2005). Interestingly, the $C W C 2$ gene was also originally discovered by an insertional mutagenesis approach with different settings. The finding of T-DNA insertion at the CWC2 locus fell into the "blind" category and also validated our suppressor screening approach.

Identification of the suppressor strain AY18 is interesting and its different filamentous phenotypes in mating and monokaryotic fruiting are even more intriguing. Although three copies of $\mathrm{T}$ DNA were found to be linked and integrate in the AY18 genome, we were able to identify the insertion site and demonstrate that the C. neoformans SSN8 homologue is responsible for the suppression phenotypes. S. cerevisiae Ssn8p is a mediator component of the RNA polymerase II holoenzyme, which negatively regulates diverse physiological processes (Björklund and Gustafsson, 2005). The SSN8 gene was originally identified as a suppressor of the snf1 mutation involving in glucose catabolite repression (Carlson et al., 1984). To reveal the mechanism leading to the restoration of mating filamentation, the transcript level of $C W C 1$ in the AY18 or YSC1 strain was recently verified by quantitative real-time PCR analysis. We found that the CWC1 gene remained at the overexpression level in these strain, suggesting the filamentation phe- notypes were not due to down-regulation of the CWC1 overexpression (results not shown). The expression study and phenotypic features of these strains suggest that $C$. neoformans Ssn8 possibly functions downstream of the Cwc complex via a more specific manner. Its dramatically derepressed monokaryotic filamentation phenotype also implies that Ssn8 might play a more critical role in the monokaryotic fruiting process.

In summary, the evolutionally conserved white collar proteins, Cwc1 and Cwc2, are two central blue regulators of $C$. neoformans. In this report, we characterized and confirmed the importance of putative domains identified in both proteins. By conducting a screen for suppressor mutants, we found genes involved in the blue light-inhibited filamentation pathways. In addition to the CWC2 and SSN8 genes, 15 other suppressor genes were identified by inverse PCR as listed in Table 3. According to the annotated information, they appear to be involved in chromatin modification, transcriptional regulation, bud site selection, meiosis and nutrient uptake and metabolism. Gene deletion of some of these genes, including the SSN8 homologue, in the wild-type background exhibited no apparent, or slightly enhanced, mating phenotypes; on the other hand, overexpression of these genes consistently showed reduced mating phenotypes (results not shown). These preliminary results support our models in which negative regulators function in the pathway. Environmental signal such as light is one of the stimuli that controls filamentous growth of $C$. neoformans. Light was also demonstrated to influence mycelial growth and colony morphology of T. borchii and N. crassa (Ambra et al., 2004). In addition to unraveling the light signaling mechanism in C. neoformans, our studies might further provide insight into how hyphal growth is regulated in fungi.

\section{Acknowledgments}

We thank Drs. Alexander Idnurm and Joseph Heitman for kindly providing the A. tumefaciens strain LBA4404 bearing the plasmid pPZP-201BK. We also thank the genome sequence information provided at Stanford Genome Technology Center, the Institute for Genome Research, the Broad Institute, and Duke University. This work is supported by National Science Council Grants NSC942311-B-002-031, NSC95-2311-B-002-010, and NSC96-2311-B002-022-MY3 to Wei-Chiang Shen.

\section{References}

Alspaugh, J.A., Perfect, J.R., Heitman, J., 1997. Cryptococcus neoformans mating and virulence are regulated by the G-protein alpha subunit GPA1 and cAMP. Genes Dev. 11, 3206-3217.

Ambra, R., Grimaldi, B., Zamboni, S., Filetici, P., Macino, G., Ballario, P., 2004. Photomorphogenesis in the hypogenous fungus Tuber borchii: isolation and characterization of Tbwc-1, the homologue of the blue-light photoreceptor of Neurospora crassa. Fungal Genet. Biol. 41, 688-697.

Ballario, P., Vittorioso, P., Magrelli, A., Talora, C., Cabibbo, A., Macino, G., 1996. White collar-1, a central regulator of blue light responses in Neurospora, is a zinc finger protein. EMBO J. 15, 1650-1657.

Björklund, S., Gustafsson, C.M., 2005. The yeast Mediator complex and its regulation. Trends Biochem. Sci. 30, 240-244.

Blumenstein, A., Vienken, K., Tasler, R., Purschwitz, J., Veith, D., Frankenberg-Dinkel, N., Fischer, R., 2005. The Aspergillus nidulans phytochrome FphA represses sexual development in red light. Curr. Biol. 15, 1833-1838.

Carlile, M.J., 1965. Photobiology of fungi. Annu. Rev. Plant Physiol. 16, 175-202.

Carlson, M., Osmond, B.C., Neigeborn, L., Botstein, D., 1984. A suppressor of snf1 mutations causes constitutive high-level invertase synthesis in yeast. Genetics 107, 19-32.

Casadevall, A., Perfect, J.R., 1998. Cryptococcus neoformans. ASM Press, Washington, DC.

Casas-Flores, S., Rios-Momberg, M., Bibbins, M., Ponce-Noyola, P., Herrera-Estrella, A., 2004. BLR-1 and BLR-2, key regulatory elements of photoconidiation and mycelial growth in Trichoderma atroviride. Microbiology 150, 3561-3569.

Corrochano, L.M., 2007. Fungal photoreceptors: sensory molecules for fungal development and behaviour. Photochem. Photobiol. Sci. 6, 725-736.

de Groot, M.J.A., Bundock, P., Hooykaas, P.J.J., Beijersbergen, A.G.M., 1998 Agrobacterium tumefaciens-mediated transformation of filamentous fungi. Nat. Biotechnol. 16, 839-842. 
Dunlap, J.C., Loros, J.J., 2006. How fungi keep time: circadian system in Neurospora and other fungi. Curr. Opin. Microbiol. 9, 579-587.

Edman, J.C., Kwon-Chung, K.J., 1990. Isolation of the URA5 gene from Cryptococcus neoformans var neoformans and its use as a selective marker for transformation. Mol. Cell. Biol. 10, 4538-4544.

Estrada, A.F., Avalos, J., 2008. The White Collar protein WcoA of Fusarium fujikuroi is not essential for photocarotenogenesis, but is involved in the regulation of secondary metabolism and conidiation. Fungal Genet. Biol. 45, 705-718.

Froehlich, A.C., Liu, Y., Loros, J.J., Dunlap, J.C., 2002. White Collar-1, a circadian blue light photoreceptor, binding to the frequency promoter. Science 297, 815-819.

Froehlich, A.C., Noh, B., Vierstra, R.D., Loros, J., Dunlap, J.C., 2005. Genetic and molecular analysis of phytochromes from the filamentous fungus Neurospora crassa. Eukaryot. Cell 4, 2140-2152.

Griffith, G.W., Jenkins, G.I., Milner-White, E.J., Clutterbuck, A.J., 1994. Homology at the amino acid level between plant phytochromes and a regulator of asexual sporulation in Emericella (=Aspergillus) nidulans. Photochem. Photobiol. 59, 252256.

Grimaldi, B., Coiro, P., Filetici, P., Berge, E., Dobosy, J.R., Freitag, M., Selker, E.U., Ballario, P., 2006. The Neurospora crassa White Collar-1 dependent blue light response requires acetylation of histone H3 lysine 14 by NGF-1. Mol. Biol. Cell 17, 4576-4583.

Guthrie, C., Fink, G.R., 1991. Guide to Yeast Genetics and Molecular Biology. Academic Press, San Diego.

He, Q., Cha, J., He, Q., Lee, H.C., Yang, Y., Liu, Y., 2006. CKI and CKII mediate the FREQUENCY-dependent phosphorylation of the WHITE COLLAR complex to close the Neurospora circadian negative feedback loop. Genes Dev. 20, 25522565.

He, Q., Cheng, P., Yang, Y., Wang, L., Gardner, K.H., Liu, Y., 2002. White collar-1, a DNA binding transcription factor and a light sensor. Science 297, 840-843.

He, Q., Liu, Y., 2005. Molecular mechanism of light responses in Neurospora: from light-induced transcription to photoadaptation. Genes Dev. 19, 2888-2899.

Herrera-Estrella, A., Horwitz, B.A., 2007. Looking through the eyes of fungi: molecular genetics of photoreception. Mol. Microbiol. 64, 5-15.

Huang, G., Chen, S., Li, S., Cha, J., Long, C., Li, L., He, Q., Liu, Y., 2007. Protein kinase A and casein kinases mediate sequential phosphorylation events in the circadian negative feedback loop. Genes Dev. 21, 3283-3295.

Hull, C.M., Heitman, J., 2002. Genetics of Cryptococcus neoformans. Annu. Rev. Genet. 36, 557-615.

Idnurm, A., Heitman, J., 2005. Light controls growth and development via a conserved pathway in the fungal kingdom. PLoS Biol. 3, e95.

Idnurm, A., Reedy, J.L., Nussbaum, J.C., Heitman, J., 2004. Cryptococcus neoformans virulence gene discovery through insertional mutagenesis. Eukaryot. Cell 3, $420-429$.

Idnurm, A., Rodriguez-Romero, J., Corrochano, L.M., Sanz, C., Iturriaga, E.A., Eslava, A.P., Heitman, J., 2006. The Phycomyces madA gene encodes a blue-light photoreceptor for phototropism and other light responses. Proc. Natl. Acad. Sci. USA 103, 4546-4551.

Kihara, J., Moriwaki, A., Tanaka, N., Ueno, M., Arase, S., 2007. Characterization of the BLR1 gene encoding a putative blue-light regulator in the phytopathogenic fungus Bipolaris oryzae. FEMS Microbiol. Lett. 266, 110-118.

Kwon-Chung, K.J., 1976. Morphogenesis of Filobasidiella neoformans, the sexual state of Cryptococcus neoformans. Mycologia 68, 821-833.

Kwon-Chung, K.J., Edman, J.C., Wickes, B.L., 1992. Genetic association of mating types and virulence in Cryptococcus neoformans. Infect. Immun. 60, 602-605.

Lee, K., Singh, P., Chung, W.C., Ash, J., Kim, T.S., Hang, L., Park, S., 2006. Light regulation of asexual development in the rice blast fungus, Magnaporthe oryzae. Fungal Genet. Biol. 43, 694-706.

Lin, X., Heitman, J., 2006. The biology of the Cryptococcus neoformans species complex. Annu. Rev. Microbiol. 60, 69-105.
Lin, X., Huang, J.C., Mitchell, T.G., Heitman, J., 2006. Virulence attributes and hyphal growth of $C$. neoformans are quantitative traits and the MATa allele enhances filamentation. PLoS Genet. 2, e187.

Lin, X., Hull, C.M., Heitman, J., 2005. Sexual reproduction between partners of the same mating type in Cryptococcus neoformans. Nature 434, 1017-1021.

Linden, H., Ballario, P., Macino, G., 1997. Blue light regulation in Neurospora crassa. Fungal Genet. Biol. 22, 141-150.

Linden, H., Macino, G., 1997. White collar 2, a partner in blue-light signal transduction, controlling expression of light-regulated genes in Neurospora crassa. EMBO J. 16, 98-109.

Liu, Y., He, Q., Cheng, P., 2003. Photoreception in Neurospora: a tale of two White Collar proteins. Cell. Mol. Life Sci. 60, 2131-2138.

Loftus, B.J., Fung, E., Roncaglia, P., Rowley, D., Amedeo, P., et al., 2005. The genome of the basidiomycetous yeast and human pathogen Cryptococcus neoformans. Science 307, 1321-1324.

Lu, Y.K., Sun, K.H., Shen, W.C., 2005. Blue light negatively regulates the sexual filamentation via the Cwc1 and Cwc2 proteins in Cryptococcus neoformans. Mol. Microbiol. 56, 480-491.

Michielse, C.B., Hooykaas, P.J., van den Hondel, C.A., Ram, A.F., 2005. Agrobacteriummediated transformation as a tool for functional genomics in fungi. Curr. Genet. $48,1-17$.

Mooney, J.L., Yager, L.N., 1990. Light is required for conidiation in Aspergillus nidulans. Genes Dev. 4, 1473-1482.

Moore, T.D., Edman, J.C., 1993. The $\alpha$-mating type locus of Cryptococcus neoformans contains a peptide pheromone gene. Mol. Cell. Biol. 13, 1962-1970.

Perfect, J.R., Toffaletti, D.L., Rude, T.H., 1993. The gene encoding phosphoribosylaminoimidazole carboxylase (ADE2) is essential for growth of Cryptococcus neoformans in cerebrospinal fluid. Infect. Immun. 61, 44464451.

Purschwitz, J., Müller, S., Kastner, C., Fischer, R., 2006. Seeing the rainbow: light sensing in fungi. Curr. Opin. Microbiol. 9, 566-571.

Purschwitz, J., Müller, S., Kastner, C., SchÖser, M., Haas, H., Espeso, E.A., Atoui, A. Calvo, A.M., Fischer, R., 2008. Functional and physical interaction of blue- and red-light sensors in Aspergillus nidulans. Curr. Biol. 18, 255-259.

Sambrook, J., Russell, I., 2001. Molecular Cloning: A Laboratory Manual. Cold Spring Harbor Laboratory Press, Cold Spring Harbor, NY.

Shen, W.-C., Davidson, R.C., Cox, G.M., Heitman, J., 2002. Pheromones stimulate mating and differentiation via paracrine and autocrine signaling in Cryptococcus neoformans. Eukaryot. Cell 1, 366-377.

Silva, F., Torres-Martinez, S., Garre, V., 2006. Distinct white collar-1 genes control specific light responses in Mucor circinelloides. Mol. Microbiol. 61, 10231037.

Tan, K.K., 1978. Light-inducing fungal development. In: Smith, J.E., Berry, D.R. (Eds.), The Filamentous Fungi, vol. 3. E. Arnold, London, pp. 334-357.

Terashima, K., Yuki, K., Muraguchi, H., Akiyama, M., Kamada, T., 2005. The dst1 gene involved in mushroom photomorphogenesis of Coprinus cinereus encodes a putative photoreceptor for blue light. Genetics 171, 101-108.

Toffaletti, D.L., Rude, T.H., Johnston, S.A., Durack, D.T., Perfect, J.R., 1993. Gene transfer in Cryptococcus neoformans by use of biolistic delivery of DNA. J. Bacteriol. 175, 1405-1411.

Walton, F.J., Idnurm, A., Heitman, J., 2005. Novel gene functions required for melanization of the human pathogen Cryptococcus neoformans. Mol. Microbiol. 57, 1381-1396.

Wang, P., Perfect, J.R., Heitman, J., 2000. The G-protein $\beta$ subunit GPB1 is required for mating and haploid fruiting in Cryptococcus neoformans. Mol. Cell. Biol. 20, 352-362.

Wickes, B.L., Mayorga, M.E., Edman, U., Edman, J.C., 1996. Dimorphism and haploid fruiting in Cryptococcus neoformans: association with the $\alpha$-mating type. Proc. Natl. Acad. Sci. USA 93, 7327-7331. 